\title{
A comparison of colour, shape, and flash induced illusory line motion
}

\author{
Jeff P. Hamm ${ }^{1}$
}

Published online: 4 January 2017

(C) The Psychonomic Society, Inc. 2017

\begin{abstract}
When a bar suddenly appears between two boxes, the bar will appear to shoot away from the box that matches it in colour or in shape - a phenomenon referred to as attribute priming of illusory line motion (ILM; colour ILM and shape ILM, respectively). If the two boxes are identical, ILM will still occur away from a box if it changes luminance shortly before the presentation of the bar (flash ILM). This flash condition has been suggested to produce the illusory motion due to the formation of an attentional gradient surrounding the flashed location. However, colour ILM and shape ILM cannot be explained by an attentional gradient as there is no way for attention to select the matching box prior to the presentation of the bar. These findings challenge the attentional gradient explanation for ILM, but only if it is assumed that ILM arises for the same underlying reason. Two experiments are presented that address the question of whether or not ${ }_{\text {flash }} \mathrm{ILM}$ is the same as colour ILM or ${ }_{\text {shape }}$ ILM. The results suggest that while colour $_{\text {ILM }}$ and shape $I L M$ reflect a common illusion, flash ILM arises for a different reason. Therefore, the attentional gradient explanation for ${ }_{\text {flash }} \mathrm{ILM}$ is not refuted by the occurrence of colour $\mathrm{ILM}$ or shape ILM, which may reflect transformational apparent motion (TAM).
\end{abstract}

Keywords Attribute priming $\cdot$ Attention priming $\cdot$ Illusory line motion · Transformational apparent motion · Individual differences

Jeff P. Hamm

j.hamm@auckland.ac.nz

1 Cognitive Neuroscience Research Group, School of Psychology, The University of Auckland, Auckland, New Zealand 1001
When a bar is presented between two boxes, the bar will appear to shoot out of both boxes and collide in the middle (Christie, 2014; Christie \& Klein, 2005). However, if one of the boxes briefly changes luminance prior to the presentation of the bar (Hamm \& Klein, 2002; Han, Zhu, Corballis, \& Hamm, 2016; Hikosaka, Miyauchi, \& Shimojo, 1993a, 1993b, 1993c; Steinman, Steinman, \& Lehmkuhle, 1995) or prior to the removal of an existing bar (Crawford et al., 2010; Hamm et al., 2014; Han et al., 2016), the bar will appear to shoot out of the flashed box, a phenomenon known as illusory line motion (flash $\mathrm{ILM}$ ).

In situations as those just described, ILM (Hamm et al., 2014; Hikosaka et al., 1993a, 1993b, 1993c; Schmidt \& Klein, 1997; Steinman et al., 1995) is thought to reflect a gradient of prior entry benefits radiating from the focus of exogenously oriented attention (Shore, Spence, \& Klein, 2001). The prior entry benefits can be offset by presenting a bar in actual motion towards the flashed location (Crawford et al., 2010; Han et al., 2016; Steinman et al., 1995). Consistent with the attentional explanation, displays that produce flash $\mathrm{ILM}$ also activate the neural networks associated with visual attention (Hamm et al., 2014). ILM has been also been shown to occur after attention has been directed by auditory or tactile cues (Shimojo, Miyauchi, \& Hikosaka, 1997) or by reflexive gaze orienting (Bavelier, Schneider, \& Monacelli, 2002), and so ILM cannot be due solely to lowlevel visual features associated with the boxes and bar. Unpublished data from this lab has shown that individuals who show large flash $I L M$ also tend to show large costs plus benefits in an attentional cuing task ( $\mathrm{Ha}, \mathrm{Li}$, Patten, \& Hamm, in press).

As outlined, the evidence for attention being capable of producing illusory motion is diverse and consistent with the understanding of how exogenous attention produces prior entry benefits (Shore et al., 2001) that are distributed as a 
gradient (Laberge, 1983). While beyond the scope of this article, it should be noted that reflective gaze ILM (Bavelier et al., 2002) may not be due to prior entry benefits as this form of shifting attention seems not to result in these benefits (Schneider \& Bavelier, 2003), but it also cannot be due to low-level visual features between the cue and the bar. Schneider and Bavelier (2003) also suggest that the sensory acceleration shown in response to exogenous cues may be due to local sensory interactions between the cue and stimulus rather than to attention. This was partly based on the finding that arrow cues did not produce evidence for prior entry; however, attention oriented by arrows is not the same attentional process as that captured by peripheral flashes (Briand \& Klein, 1987). In addition, Schneider and Bavelier (2003) found that sensory acceleration occurred at multiple cue locations, and argued that exogenous attention cannot be distributed to so many locations and suggested that the prior entry effects are therefore not due to attention. As ILM is thought to reflect preattention- and attentional-based influences (von Grünau, Dube, \& Kwas, 1996), it may be that the multiple cues of Schneider and Bavelier (2003) are reflecting the preattentive aspects with attention contributing on top of this. Schneider and Bavelier (2003) allow for the possibility that attention could contribute a small prior entry benefit as well. Despite the debate over what process is responsible for the sensory facilitation, the critical notion is that a peripheral onset cue results in a gradient of this sensory facilitation.

Furthermore, it is accepted that attention is unlikely to be the only process that results in ILM and it may be that reflexive gaze ILM is also indicative of a different class of illusion. There appear to be preattentive low-level mechanisms that also produce ILM (Jancke, Chavane, Naarman, \& Girinvald, 2004; von Grünau et al., 1996) as well as mechanisms that result in motion due to matching attributes between successive stimuli (Corballis, Funnell, \& Gazzaniga, 2002; Tse, Cavanagh, \& Nakayama, 1998; von Grünau \& Faubert, 1994). For example, von Grünau and Faubert (1994) have demonstrated that if two boxes of similar luminance but different colours are joined by a bar that matches in colour to one of the boxes, then the bar will appear to shoot out of the matching box. Illusions of motion that occur in this situation are referred to here as colour ILM. ColourILM is an example of attribute priming (von Grünau \& Faubert, 1994) or transformational apparent motion (TAM; Tse et al., 1998). Because the bar is equally likely to match either colour the participant cannot anticipate which box the bar will match prior to the onset of the bar. A bias in the location of attention prior to the onset of the line is a necessary condition in the attentional gradient explanation.

It has been argued that because attention cannot explain the illusions of motion in some display configurations that the attentional gradient theory must be rejected. For example, in Jancke et al. (2004) subcortical cellular activity in visual cortex of anaesthetised cats was indistinguishable between real motion and illusory motion. Even though it was not demonstrated that this activity was associated with actual motion perception, and disregarding the fact that a different species is under investigation, the conclusion from Jancke et al. (2004) was that a bottom-up mechanism underlying the line-motion illusion, without need of attention, was demonstrated. Inherently, though, to draw this conclusion requires accepting the assumption that the polarized gamma motion (Kanizsa, 1979) that occurs in the single box and bar display arises for the same underlying reason as the ILM that occurs in the two-box and flash paradigm. The assumption that there is only one way that ILM can be produced appears unwarranted (Han et al., 2016; von Grünau et al., 1996), and so there is no reason to reject the attentional gradient hypothesis simply because another illusion can be explained by low-level cellular activity.

More generally, if there are multiple routes to ILM, then demonstrating an illusion of motion in two paradigms, one of which cannot be explained by a given theory, is not by itself a refutation of that explanation with respect to the illusion arising in the other paradigm. Han et al. (2016) argued that if two conditions generate an illusion of motion for the same reason, then participants who show a large illusion in one case will show a large illusion in the other; the illusions will be correlated at the individual participant level.

It seems clear that ILM that arises through attribute priming cannot be explained by endogenous or exogenous visual attention. And while endogenous attention has been argued to be ineffective in generating ILM (Christie, 2014; Christie \& Klein, 2005), plenty of evidence suggests that attention can generate ${ }_{\text {flash }}$ ILM. Moreover, the fact that ILM can be generated by nonvisual cues of attention (Shimojo et al., 1997) seems impossible to explain via attribute priming. Furthermore, different neural networks are activated by flash ILM (Hamm et al., 2014) and colour ILM (Tse, 2006), with the former consistent with a role for attention and the latter suggesting the influence of object processing. Therefore, there is plenty of evidence to suggest that colour $\mathrm{ILM}$ and ${ }_{\text {flash }} \mathrm{ILM}$ may not be the same illusion, meaning the two displays employed appear to produce illusory motion for different underlying reasons. If ${ }_{\text {flash }} \mathrm{ILM}$ arises for different reasons from colour ILM, then there should be no relationship between a participant's ${ }_{\text {flash }}$ ILM and their colour ILM.

To correlate the illusions, Han et al. (2016) recommend quantifying ILM as the area between the percept curves following a left and right flash, because this quantity does not require the fitting of a psychometric function, but is calculated by summing the area of trapezoids directly based upon the observed percept scores. This avoids the issue of introducing another level of methodological variation, because what function is used to model the behaviour, and what criterion is used to determine if a given participant's data is well modelled by 
that function, can vary study to study. It is this exactly this kind of variation between studies in terms of the analysis and experimental protocols that is argued to be particularly problematic in this area of research in the first place (see Han et al., 2016 for a discussion on how subtle changes in display parameters can result in the observance of ILM for different and unrelated reasons). For colour $\mathrm{ILM}$, the area between the curves is measured as the area under the curve away from the box whose colour matches the bar when the box is on the left side minus the area under the curve when the matching box is on the right side. Han et al. (2016) also recommend that correlations should be assessed only after the removal of data with a large Cook's distance value (Cohen \& Cohen, 1983), because these points exert sufficient influence on the correlation as to be considered nonrepresentative. The same approach will be employed here to maintain a much-needed level of consistency in the literature. Therefore, the purpose of Experiment 1 was to test whether or not ${ }_{\text {flash }}$ ILM was related to ${ }_{\text {colour }} \mathrm{ILM}$ by measuring ILM $\mathrm{Irea}_{\text {are }}$ for both and determining if these values were correlated. The study is also a test of the utility of the individual differences approach to testing whether or not two illusions are likely to arise for the same reason (Han et al., 2016) in that the various conditions that produce flash ILM or colourILM should be correlated, but the relationships between flash $_{\text {ILM and }}$ colour ILM should not be correlated.

\section{Experiment 1}

\section{Method}

\section{Subjects}

Twenty-five students recruited from the University of Auckland student body volunteered to participant in this study. One female participant was dropped from the study because the experimental software failed to record her responses, and so the data presented is from 24 participants (seven males and 17 females; $M_{\text {age }}=21.2$ years, $S D=1.98$ years). All reported normal or corrected-to-normal vision, and 21 were right-handed, as assessed by a laterality score greater than zero $(M=82.3)$; two were left-handed with scores less than zero $(M=-58.9)^{\prime}$ and one had a laterality score of zero exactly on the Edinburgh Inventory (Oldfield, 1971). All methods were approved by the University of Auckland's Human Participants Ethics Committee.

\section{Apparatus}

Stimuli were presented on a SVGA CRT $60 \mathrm{~Hz}$ monitor at a resolution of $600 \times 480$ pixels. Stimulus presentation and timing routines were control by a Pentium II, $500 \mathrm{MHz}$ computer with an $\mathrm{S} 3$ video card running Windows 98 rebooted in
Dos Mode to ensure the accuracy of the millisecond timing routines (Myors, 1999). The experimental software was written in Borland Pascal 7.0 and employed timing routines found in Hamm (2001), with synchronization with the raster scan achieved as described by Heathcote (1988). The luminance of the stimuli were measured with a Konica Minolta LS-100 luminance meter and are based on the mean of five readings taken from the approximate middle of the CRT screen when it was set to be entirely the tested colour.

\section{Stimuli}

The background was a neutral grey $\left(13.07 \mathrm{~cd} / \mathrm{m}^{2}\right)$. The fixation cross was presented in black $\left(4.04 \mathrm{~cd} / \mathrm{m}^{2}\right)$ in the centre of the display and measured $0.53^{\circ} \times 0.53^{\circ}$. The two boxes measured $1.96^{\circ} \times 1.96^{\circ}$, and were separated by $9.60^{\circ}$ centre to centre. The left and right boxes' centres were equally shifted left and right from the fixation cross and were $1.19^{\circ}$ above the centre of the fixation cross. The bar that joined the boxes touched the edge of both boxes and was $1.48^{\circ}$ in height and centred with the boxes. The display details are presented in Fig. 1a.

\section{Procedure}

Minimizing luminance differences The perceptual luminance of display colours was achieved using software
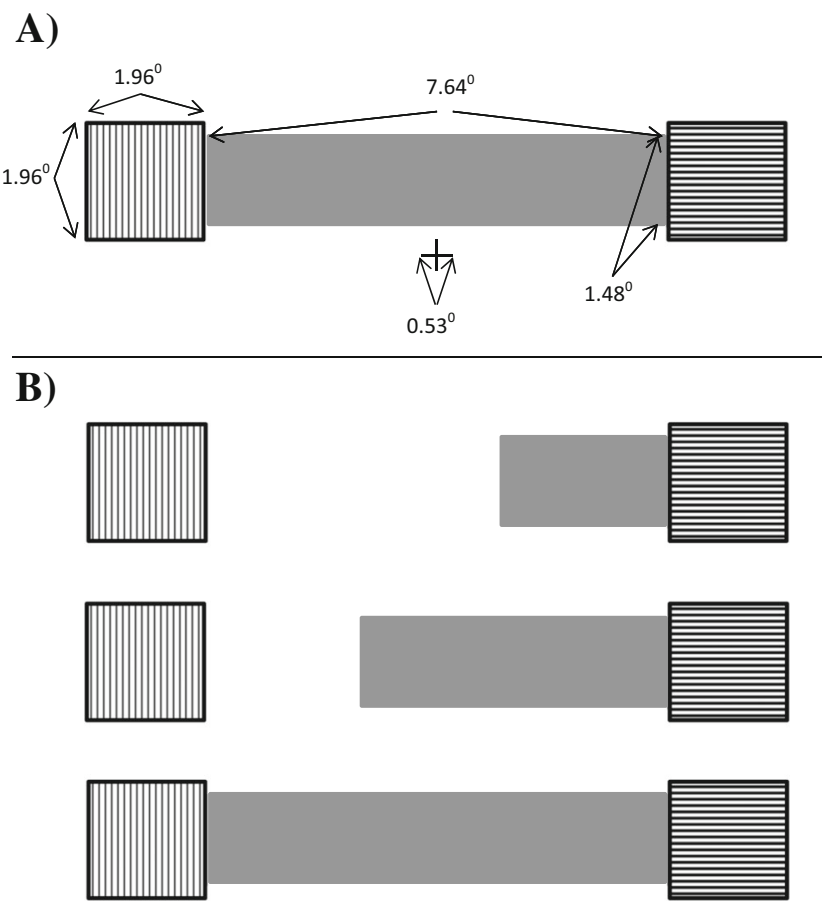

Fig. 1 a Display after the full presentation of the bar. Vertical and horizontal bars are used to represent the fact that the two boxes of are different hues (one red, one green) while the solid bar indicates it could match the left, right, or neither box. b Depiction of real motion to the left at medium speed, spanning three screen refreshes 
procedures that enabled a process similar to those described by Troscianko and Low (1986). The experiment began by presenting a red square measuring $4.82^{\circ} \times 4.82^{\circ}$ and 7.34 $\mathrm{cd} / \mathrm{m}^{2}$ in the middle of a bright green background $(38.24 \mathrm{~cd} /$ $\mathrm{m}^{2}$ ). The colour of the square and background were swapped every three screen refreshes, $50 \mathrm{~ms}$, and the participant was asked to make adjustments using the number pad to minimize the flickering. The 4 and 5 key could be used to make large adjustments that increased or decreased the RGB value by 5 , while the 1 and 2 keys were used to make $+/-1$ adjustments to the RGB value. During phase one of the luminance setting, the luminance of red was kept constant at $\left(7.85 \mathrm{~cd} / \mathrm{m}^{2}\right)$, which corresponded to an RGB setting of 40 . The participant adjusted the RGB setting for green until they decided the flickering was minimized. The mean luminance for green was $7.34 \mathrm{~cd} /$ $\mathrm{m}^{2}(\mathrm{RGB}=23.6)$, with a standard deviation of $0.71 \mathrm{~cd} / \mathrm{m}^{2}$. After minimizing the flicker between red and green they hit enter to move to Phase 2. At this point, a third colour, grey, was included, and the colour of the square and background flickered such that the grey was always present and cycled between the central square and background, while red and green were each cycled through the alternate portion of the display (i.e. grey background and green square $\rightarrow$ green background and grey square $\rightarrow$ grey background and red square $\rightarrow$ red background and grey square $\rightarrow$ repeat). Pressing the keys adjusted the level of brightness for the grey only, while the red and green were maintained at the levels determined in Phase 1. The luminance of the grey was then adjusted to minimise the flicker between the three colours. The mean luminance for grey was $7.55 \mathrm{~cd} / \mathrm{m}^{2}(\mathrm{RGB}=19.4)$ with a standard deviation of $0.68 \mathrm{~cd} / \mathrm{m}^{2}$. The luminance levels determined from phase one and two were used for the starting colour of the boxes and for the presentation of the red, green, and grey bars.

The participant hit enter to begin Phase 3 to equate the luminance values of the red and green flashes. Phase 3 involved only the colours red and green, where green was adjusted to minimize the flicker with red as in Phase 1, however, red was set at a constant luminance of $13.99 \mathrm{~cd} / \mathrm{m}^{2}(\mathrm{RGB}=$ 63). Green was adjusted to a mean setting of $13.84 \mathrm{~cd} / \mathrm{m}^{2}$ $(\mathrm{RGB}=36.4)$ with a standard deviation of $3.19 \mathrm{~cd} / \mathrm{m}^{2}$. The level from Phase 3 were used for the flashes during the flash ILM trials.

A trial began with the presentation of the fixation cross for $500 \mathrm{~ms}$, after which the two boxes were presented. On half the trials, the box on the left was red and the box on the right was green, while the colours were reversed on the remaining half of the trials. After a 500-ms delay, either the left, right, or neither of the two boxes increased in luminance for $50 \mathrm{~ms}$ and then returned to the initial luminance level. On the same screen that the flash ended, the presentation of the bar began. It was intended that the bar was to be either presented in its entirety on a single screen refresh (no real motion), or during real motion trials it was to be presented unfolding over two, three, or four screen refreshes by presenting the bar in successive halves, thirds, or quarters. to produce fast, medium, or slow real motion to the left or right (see Fig. $1 \mathrm{~b}$ for a depiction of leftward real motion over three screens). However due to a programming error, all motion conditions were reduced by one, resulting in the two fast-motion conditions replicating the no-motion condition and no trials occurring in the foursegment (slow) conditions. The colour of the bar (3; red, green, grey), the arrangement of the coloured boxes (2; red/ green and green/red), location of the flash ( 3 ; left/right/none), and the intended real motion conditions ( 7 ; slow left, medium left, fast left, no motion, fast right, medium right, slow right) were all completely counterbalanced; however, after completion of data collection, it was noted that the real motion contained only five conditions (medium left, fast left, no motion [ $\mathrm{x} 3$, fast right, medium right), and the trials were recoded appropriately. For analysis, the box and bar colours were also recoded from red or green to be in terms of the bar matching the colour of the box on the left, right, or neither. Half the participants were required to respond by indicating which direction the bar appeared to move, meaning at which box the motion terminated, and half were to respond by indicating at which box the motion began; where the bar went or where the bar came from.

Responses that indicated the bar moved to the left (or came from the right) were scored as -1 , and responses that indicated the bar moved to the right (or came from the left) were scored as +1 . Any trial responded to faster than $200 \mathrm{~ms}(0.83 \%)$ were deemed to be anticipations and trials responded to longer than $2,000 \mathrm{~ms}(2.70 \%)$ were deemed to be distractions were discarded. Also discarded were any trials in which no response $(1.11 \%)$ was made after $4,000 \mathrm{~ms}$. The average of the remaining "percept scores" and decision times (based upon $95.36 \%$ of all trials) were then calculated for statistical analysis.

\section{Results}

Percept scores were calculated on the basis of the colour of the line matching the left box, right box, or neither box; the location of the flash (left, right, or neither box); and the amount of real motion in the display (five levels; medium left, fast left, no motion, fast right, medium right). Given the programming error, this resulted in 10 trials in each of the conditions with medium or fast motion, and 30 trials in each of the no motion conditions, for a total of 630 trials. The mean percept scores, arranged to show ${ }_{\text {flash }} \mathrm{ILM}$, can be seen in Figs. 2a-c, while the same data are rearranged to emphasise the colourILM in Figs. $2 \mathrm{~d}-\mathrm{f}$. In both series, ILM is quantified as the area between the left (filled squares) and right (open circles) curves. Interpretations of the statistical analysis will primarily be guided by assessing the accuracy of the predictions derived from the assumption of no effect (null hypothesis significance testing). However, when examining the correlational data, 

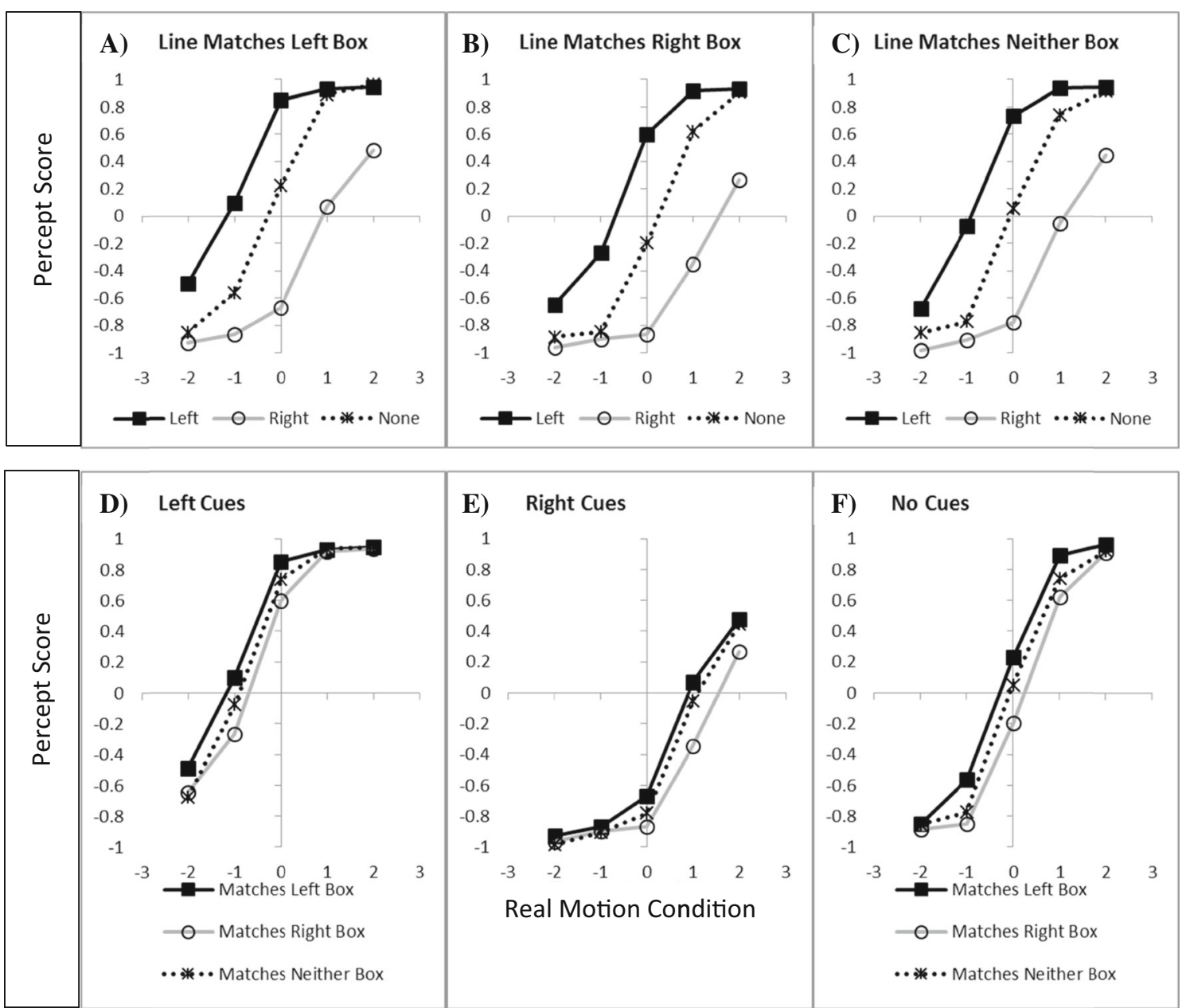

Fig. 2 Upper panels depict the group mean percept scores controlling for any influence of colour ILM; lower panels depict same data rearranged to control for ${ }_{\text {flash }}$ ILM. Left column depicts controlling of a left side inducer; middle column depicts controlling of a right side inducer; right column indicates no additional inducer and so shows pure ${ }_{\text {flash }} \mathrm{ILM}$ (upper right) and pure colour $_{\text {ILM (lower right). ILM }}$ area reflects space between left (solid squares black lines) and right (open circles grey lines) side flashes (upper row) or matching colour box (lower row) because of the theoretical importance of asserting no relationship, and given the concerns associated with accepting a null finding based upon null hypothesis significance testing, correlations will also be assessed using a Bayesian approach by calculating the probability of the null given the data $\left(\mathrm{p}_{\mathrm{H} 0 \mid \mathrm{D}}\right.$; Masson, 2011). In short, the standard $p$ value is an assessment of the accuracy of the null hypothesis to predict the observed data - if the probability of the data is low $(p<.05)$, then the null hypothesis is deemed to be inaccurate. The Bayes $\mathrm{p}_{\mathrm{HO} \mid \mathrm{D}}$ is an assessment of the relative probabilities for the null and the alternative hypotheses given the observed data. As $\mathrm{p}_{\mathrm{H} 0 \mid \mathrm{D}}$ moves away from 0.5 , where both the null and the alternative are considered equally supported by the data, then as $\mathrm{p}_{\mathrm{H} 0 \mid \mathrm{D}}$ approaches 1.0 , the null becomes increasingly probable, and as $\mathrm{p}_{\mathrm{H} 0 \mid \mathrm{D}}$ approaches 0.0 , the alternative hypothesis becomes more probable. $\mathrm{P}_{\mathrm{HO} \mid \mathrm{D}}$ is, therefore, an estimate of the probability of the null hypothesis relative to the alternative after having considered the experimental data, while the standard $p$ values is an assessment of the observed data's likelihood when predicting no effect before the data are collected. Both approaches increase our understanding through different routes, with NHST testing the accuracy of a theory while Bayesian approaches test the relative posterior probabilities of the competing hypotheses. While Popper (1963) argued that increased predictive accuracy paradoxically leads to decreased probability of being true, and that preference should be given to prediction, here it is taken that the two statistical approaches should be viewed as complimentary rather than as competing techniques.

The Bayes $\mathrm{p}_{\mathrm{H} 0 \mid \mathrm{D}}$ value will be described using terminology suggested by Raftery (see Table 6 in Raftery, 1995), where weak evidence in favour of the null is indicated by $\mathrm{p}_{\mathrm{H} 0 \mid \mathrm{D}}$ between 0.5 and 0.75 , positive evidence in favour of the null is indicated by $\mathrm{p}_{\mathrm{H} 0 \mid \mathrm{D}}$ between $0.75^{+}$and 0.95 , strong evidence in favour of the null is indicated by $\mathrm{p}_{\mathrm{H} 0 \mid \mathrm{D}}$ between $0.95^{+}$and 0.99 , and very strong evidence in favour of the null is indicated by $\mathrm{p}_{\mathrm{H} 0 \mid \mathrm{D}}>0.99$. To avoid confusion by switching between $\mathrm{p}_{\mathrm{H} 0 \mid \mathrm{D}}$ and $\mathrm{p}_{\mathrm{H} 1 \mid \mathrm{D}}$, even when evidence is in favour of the 
alternative hypothesis, the Bayesian probability will be described with reference to the probability of the null hypothesis. Therefore, weak evidence against the null is indicated by $\mathrm{p}_{\mathrm{H} 0 \mid \mathrm{D}}$ values between $0.50^{-}$and 0.25 , positive evidence against the null is indicated by $\mathrm{p}_{\mathrm{H} 0 \mid \mathrm{D}}$ values between $0.25^{-}$ and 0.05 , strong evidence against the null is indicated by $\mathrm{p}_{\mathrm{H} 0 \mid \mathrm{D}}$ values between $0.05^{-}$and 0.01 , and very strong evidence against the null is indicated by $\mathrm{p}_{\mathrm{HO} \mid \mathrm{D}}<0.01$.

The area between the left and right flash conditions was significantly different from zero when the bar matched the left, $t(23)=15.44, p<.001, M=3.80, S D=1.21)$; right, $t(23)=$ $15.46, p<.001, M=3.85, S D=1.22$; and neither box, $t(23)=$ $16.14, p<.001, M=3.74, S D=1.13$, indicating that ${ }_{\text {flash }} \mathrm{ILM}$ occurred in all conditions. These were compared in a one-way within-subject's ANOVA and there was no main effect of condition, $F(2,46)=0.303, M S E=0.263, p=.740, \eta_{\mathrm{p}}^{2}=$ 0.013 . The area between the curves when the line colour matched the left box or the right box was significantly different from zero when the flash occurred on the left, $t(23)=5.13$, $p<.001, M=0.72, S D=0.68$; right, $t(23)=4.24, p<.001, M$ $=0.77, S D=0.89$; and neither side, $t(23)=6.04, p<.001, M=$ $1.03, S D=0.83$, indicating that ${ }_{\text {colour }} \mathrm{ILM}$ occurred in all conditions. These were compared in a one-way within-subject's ANOVA, and there was no main effect of condition, $F(2,46)$ $=2.054, M S E=0.325, p=.140, \eta_{\mathrm{p}}^{2}=0.082$.

The area for the pure flash ILM and pure colourILM were compared between those who responded by indicating which box the bar appeared to move towards and those who responded by indicating which box the bar appeared to come from, with the means shown in Fig. 3. This resulted in a main effect of illusion type, $F(1,22)=91.29, M S E=0.965, p<$ $.001, \eta_{\mathrm{p}}{ }^{2}=0.806 ; M_{\text {flash }}=3.74$ versus $M_{\text {colour }}=1.03$; a main effect of group, $F(1,22)=5.73, M S E=0.877, p=.026, \eta_{\mathrm{p}}{ }^{2}=$ $0.207 ; M_{\text {from }}=2.71$ versus $M_{\mathrm{to}}=2.06$, with no interaction, $F(1,22)=0.16, M S E=0.965, p=.693, \eta_{\mathrm{p}}{ }^{2}=0.001$; $M_{\text {FlashFrom-FlashTo }}=0.68$ versus $M_{\text {ColourFrom-ColourTo }}=0.61$.

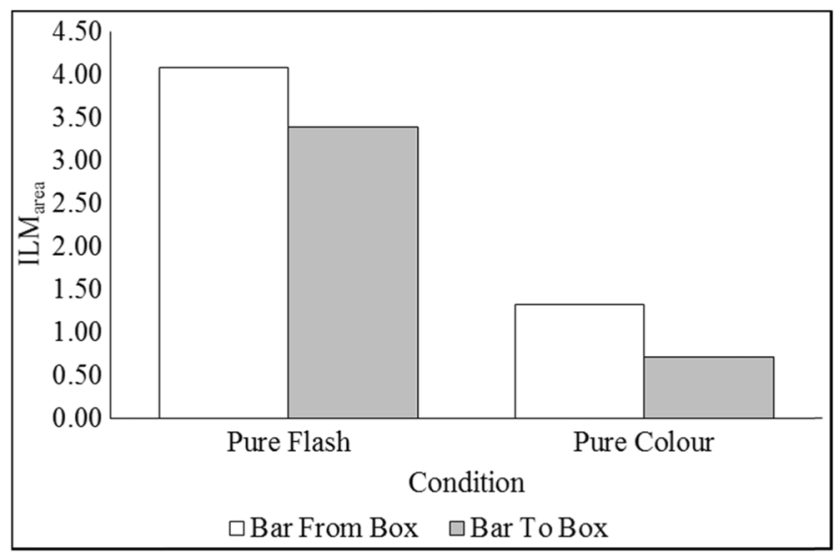

Fig. 3 Mean flash $\mathrm{ILM}_{\text {area }}$ and colour $\mathrm{ILM}_{\text {area }}$ for participants who indicated the box from which the bar motion began (open bars) and who indicated the box to which the bar motion ended (filled bars)
For the correlational analysis, the inclusion criterion a Cook's distance $<=0.1818(4 /(n-k-1)$, where $n=24$ and $k=1$; Cohen \& Cohen, 1983). As shown in Fig. 4a, an individual's area for flash ILM when the bar colour matched the left box was related, $r^{2}(21)=.5334, p<.001, \mathrm{p}_{\mathrm{H} 0 \mid \mathrm{D}}<0.001$, very strong evidence against the null hypothesis, to their area for ${ }_{\text {flash }} \mathrm{ILM}$ when the bar matched the right box, demonstrating that ${ }_{\text {flash }} \mathrm{ILM}$ has good

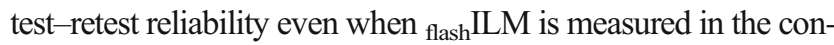
text of colour ILM. These areas were averaged and correlated against the area measure when the bar colour matched neither box, the pure ${ }_{\text {flash }} \mathrm{ILM}$ condition, and again there was good testretest reliability, $r^{2}(21)=.7972, p<.001, \mathrm{p}_{\mathrm{H} 0 \mid \mathrm{D}}<0.001$, very strong evidence against the null hypothesis, as shown in Fig. $4 \mathrm{~b}$. When the points with Cook's distance exceeding the inclusion criterion are not excluded, the relationship is $r^{2}(22)=.5789$ and .8087 , respectively.

As shown in Fig. 4c, there was not a relationship between colour ILM when the left box flashed with colour ILM when the right box flashed, $r^{2}(20)=.1272, p=.103, \mathrm{p}_{\mathrm{H} 0 \mid \mathrm{D}}=0.5122$, weak evidence in favour of the null hypothesis, but the Bayesian $\mathrm{p}_{\mathrm{HO} \mid \mathrm{D}}$ indicates the data are effectively equally supportive of a relationship and of independence and so is considered equivocal. Given the ambiguity of this initial analysis the same procedure was followed as with flash $\mathrm{ILM}$, and the areas were averaged and correlated against the area measure when neither box flashed, the pure colourILM condition. This showed there was good testretest reliability, $r^{2}(20)=.2317, p=.023, \mathrm{p}_{\mathrm{H} 0 \mid \mathrm{D}}=0.2052$, positive evidence against the null hypothesis, as shown in Fig. 4d. When the points that had Cook's distance that exceeded the inclusion criterion were not excluded the correlations were $r^{2}(22)=.2058$ and .3907 , respectively.

The area for pure versions of flash $_{1} \mathrm{ILM}$ and colour $\mathrm{ILM}$, shown in Fig. 4e, were not found to be related, $r^{2}(20)=.0003, p=$ $.939, \mathrm{p}_{\mathrm{HO} \mid \mathrm{D}}=0.817$, positive evidence in favour of the null hypothesis. There was also no relationship when flash $\mathrm{ILM}$ and colour ILM areas calculated from the mixed conditions were correlated, $r^{2}(20)=.0009, p=.895, \mathrm{p}_{\mathrm{H} 0 \mid \mathrm{D}}=0.816$, positive evidence in favour of the null hypothesis,)as shown in Fig. $4 \mathrm{f}$. When the points with Cook's distance exceeding the inclusion criterion are not excluded, the correlations were $r^{2}(22)=.005$ and .028 , respectively.

\section{Decision times}

The mean decision times are shown in Fig. 5, with the upper three panels showing the data arranged to show flash ILM, while the bottom three panels show the same data arranged according to colour ILM grouping.

The decision times were plotted as a function of the distance from the point of subjective equality. The PSE was determined by least squares fitting of the equation $2\left(\mathrm{e}^{\mathrm{ax}+\mathrm{b}} /\left(\mathrm{e}^{\mathrm{ax}+}\right.\right.$ $\left.{ }^{b}+1\right)-1$ (Han et al., 2016) to the percept scores, which is fitting a logistic regression equation scaled to be the percept 


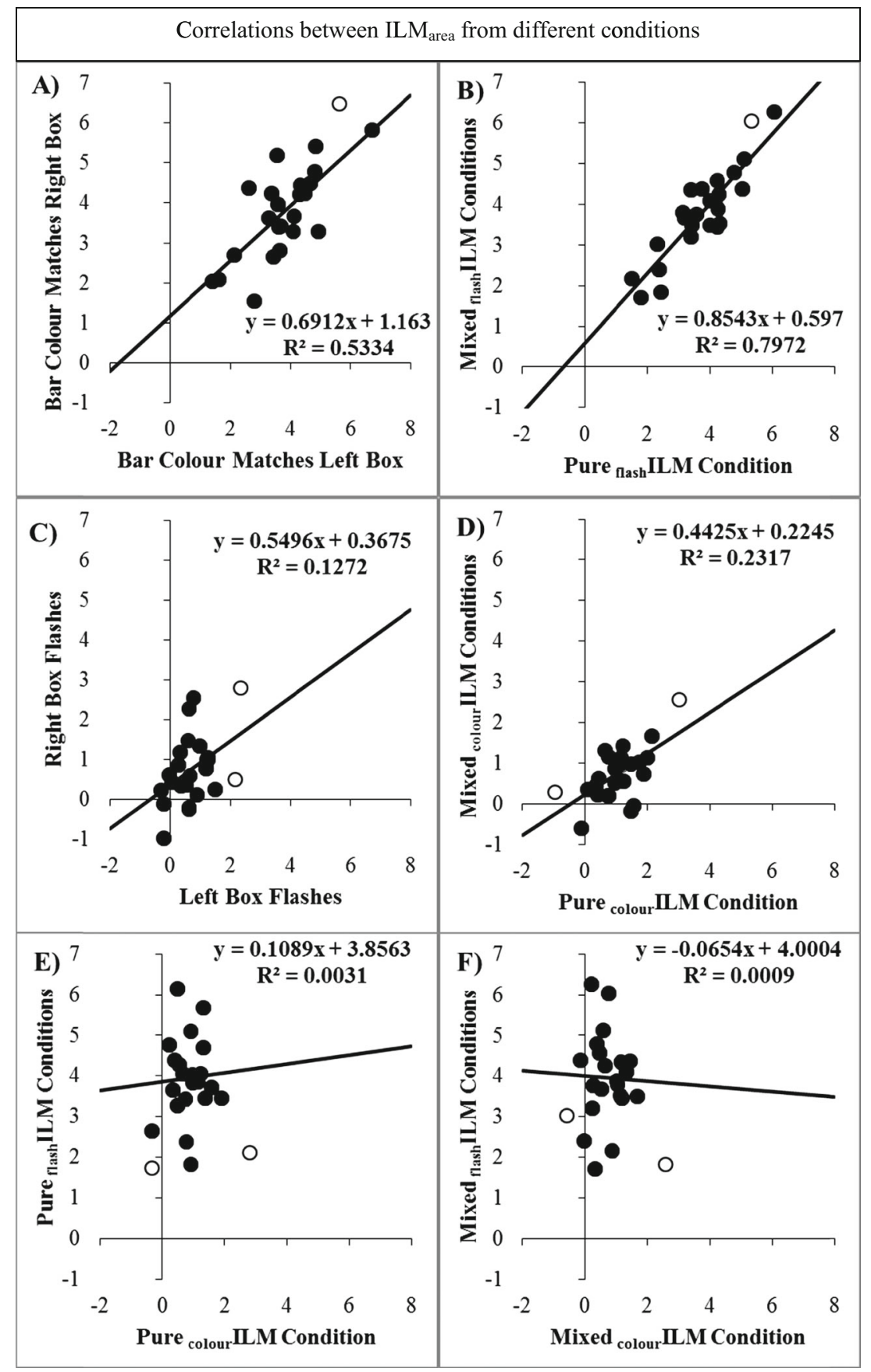

Fig. 4 Correlations of ILM area $_{\text {between }} a_{\text {flash }}$ ILM in context of left colour ILM and flash ILM in context of right colour ILM; b mixed flash ILM conditions and pure flash ILM condition; $\mathbf{c}_{\text {colour }}$ ILM $_{\text {area }}$ when in the context of left flash $\mathrm{ILM}$ and colour $\mathrm{ILM}_{\text {area }}$ when in the context of right flash ILM; $\mathbf{d}$ between mixed colour ILM conditions and pure colourILM

range of -1 to +1 . The PSE is then determined as being $-\mathrm{b} / \mathrm{a}$. The decision times that did not exceed the inclusion criterion (for the decay function the criterion was a Cook's distance $<=$ $0.0952 ; 4 /(n-k-1)$ where $n=45$ and $k=2)$ were well described by a distance decay function, as shown in Fig. 6. If the data point with the extreme Cook's distance value, indicated by the open symbol, is included the equation is condition; e between pure ${ }_{\text {flash }}$ ILM and pure colour ILM conditions; $\mathbf{f}$ between mixed flash ILM and mixed colourILM conditions. Each data point represents a participant, with open symbols indicating participants dropped from the analysis because they exceeded the Cook's distance criterion

$456.0286+308.2481 \mathrm{e}^{(-0.4467 \mathrm{x})}$, with an $r^{2}=0.8095, \mathrm{p}_{\mathrm{H} 0 \mid \mathrm{D}}<$ 0.001 , very strong evidence against the null hypothesis.

\section{Decision time congruency effect}

The decision time congruency effect is the difference between the mean decision times of conditions where the 


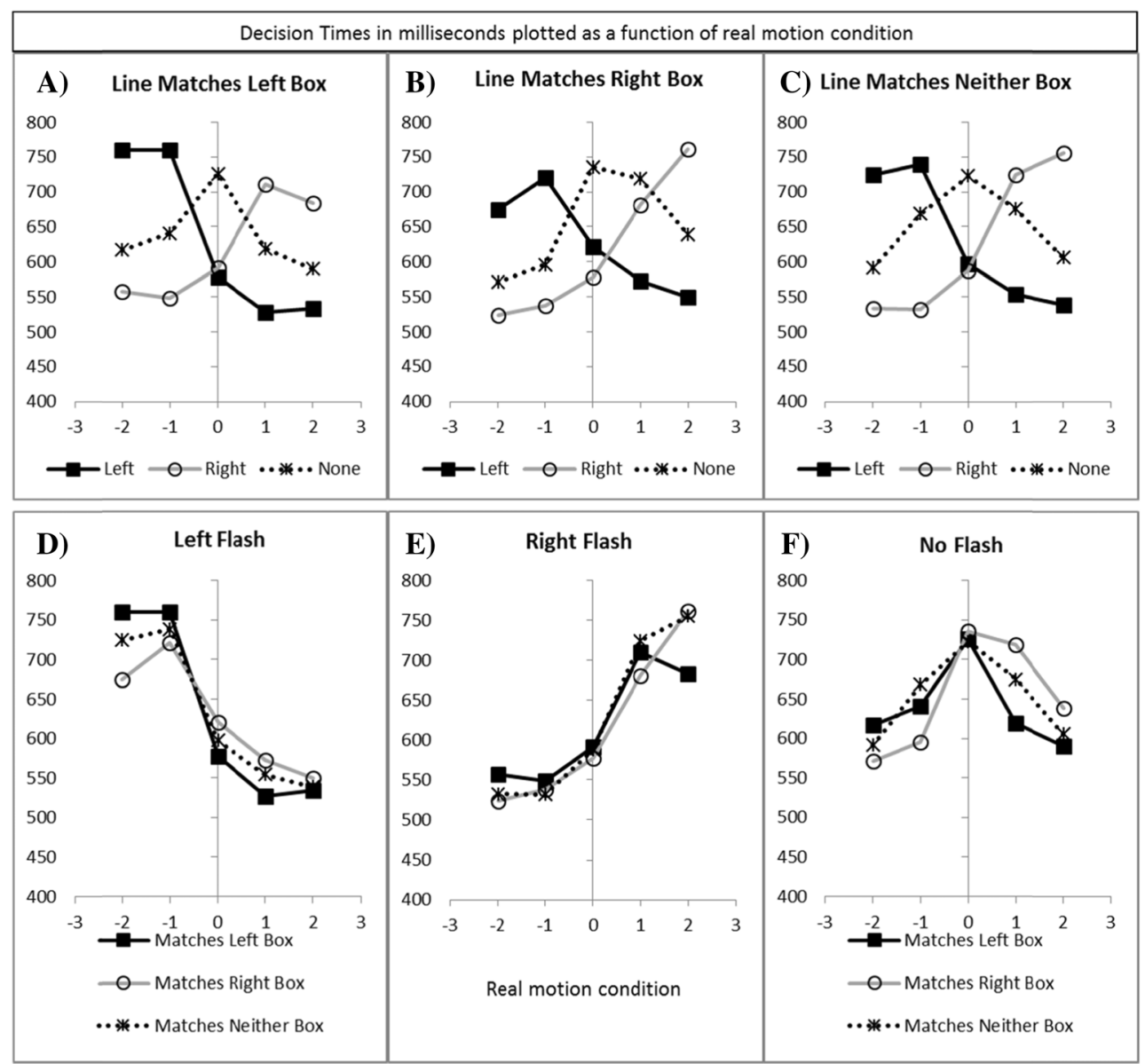

Fig. 5 Upper panels depict group mean decision times as function of inducer location and real motion when controlling for colour ILM; lower panels depict same data rearranged to control for ${ }_{\text {flash }} \mathrm{ILM}$

real motion and the illusory motion conflict and when the motions are in the same direction. This means for flash $\mathrm{ILM}$, the congruency effect is the mean of the left motion trials when the right box flashes and right motion trials when the left box flashes is subtracted from the mean of left motion trials where the left box flashes and

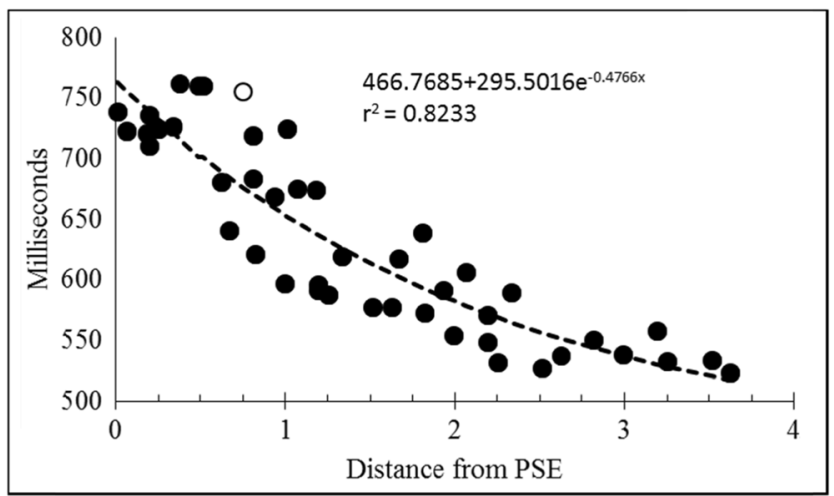

Fig. 6 Group mean decision times as a function of their distance from the mean points of subjective equality. Open symbols indicate data points dropped from analysis because they exceeded Cook's distance criterion right motion trials where the right box flashes. The mean flash ILM congruency effect was 187, 163, and $197 \mathrm{~ms}$ for when the bar colour matched the left, right, and neither box, respectively. These were analysed in a one-way ANOVA and were not found to significantly differ, $F(2$, 46) $=1.244, p=.298, M S E=5,679.574, \eta^{2}=0.051$. When the data are correspondingly arranged as per colour ILM, the mean congruency effect was 47,23 , and $60 \mathrm{~ms}$ for when the left, right, or neither box flashed, respectively. A one-way repeated-measures ANOVA did not reveal any difference, $F(2,46)=1.279, p=.288, M S E$ $=6,516.171, \eta^{2}=0.053$.

The congruency effects for the different conditions were correlated with each other, and after removing data pairs with excessive Cook's distance values, the flashbased congruency effect when the bar matched the left box was shown to be related to the congruency effect when the bar matched the right box, $r^{2}(21)=.1908, p=$ $.037, \mathrm{p}_{\mathrm{H} 0 \mid \mathrm{D}}=0.2959$, but not when the bar matched neither box, $r^{2}(20)=.1677, p=.058, \mathrm{p}_{\mathrm{H} 0 \mid \mathrm{D}}=0.3838$, although both are considered weak evidence against the null hypothesis. The congruency effect when the bar matched 
the right box was related to the congruency effect when the bar matched neither box, $r^{2}(21)=.4182, p<.001$, $\mathrm{p}_{\mathrm{H} 0 \mid \mathrm{D}}=0.0094$, which is very strong evidence against the null hypothesis. When the colourILM congruency effects were compared in the corresponding order as above, none of the relationships approached significance, $r^{2}(19)$ $=.1170, p=.129, \mathrm{p}_{\mathrm{H} 0 \mid \mathrm{D}}=0.5536$, weak evidence in favour of the null hypothesis; $r^{2}(20)=.0025, p=.824$, $\mathrm{p}_{\mathrm{HO} \mid \mathrm{D}}=0.8202$, positive evidence in favour of the null hypothesis; $r^{2}(20)=.0145, p=.5939, \mathrm{p}_{\mathrm{H} 0 \mid \mathrm{D}}=0.7998$, positive evidence in favour of the null hypothesis. When the average congruency effect for flash ILM was correlated with the average congruency effect for colourILM, no relationship was found, $r^{2}(20)=.0052, p=.749, \mathrm{p}_{\mathrm{H} 0 \mid \mathrm{D}}=$ 0.8158 , positive evidence in favour of the null hypothesis.
The scatterplots for these comparisons may be found in Figs. 7a-g.

Finally, the mean decision time congruency effect for flash ILM and colour ILM were correlated with the corresponding mean ILM $_{\text {area }}$ (see Figs. $7 \mathrm{~h}-\mathrm{i}$ ), and it was found that after removing data that exceeded the Cook's distance criterion, an individual's decision time congruency effect did not correlate significantly with their ILM $_{\text {area }}$ for $_{\text {flash }} \mathrm{ILM}, r(22)=.4014$, $p=.052, \mathrm{p}_{\mathrm{H} 0 \mid \mathrm{D}}=0.3730$, weak evidence against the null hypothesis, but the relationship was found for colourILM, $r(21)=.5770, p=.004, \mathrm{p}_{\mathrm{H} 0 \mid \mathrm{D}}=0.0436$, strong evidence against the null hypothesis. A comparison of these correlations using Fisher's $z$ transformation found no difference between the relationships $(z=1.67, p=.095)$, ruling out the implied interaction.

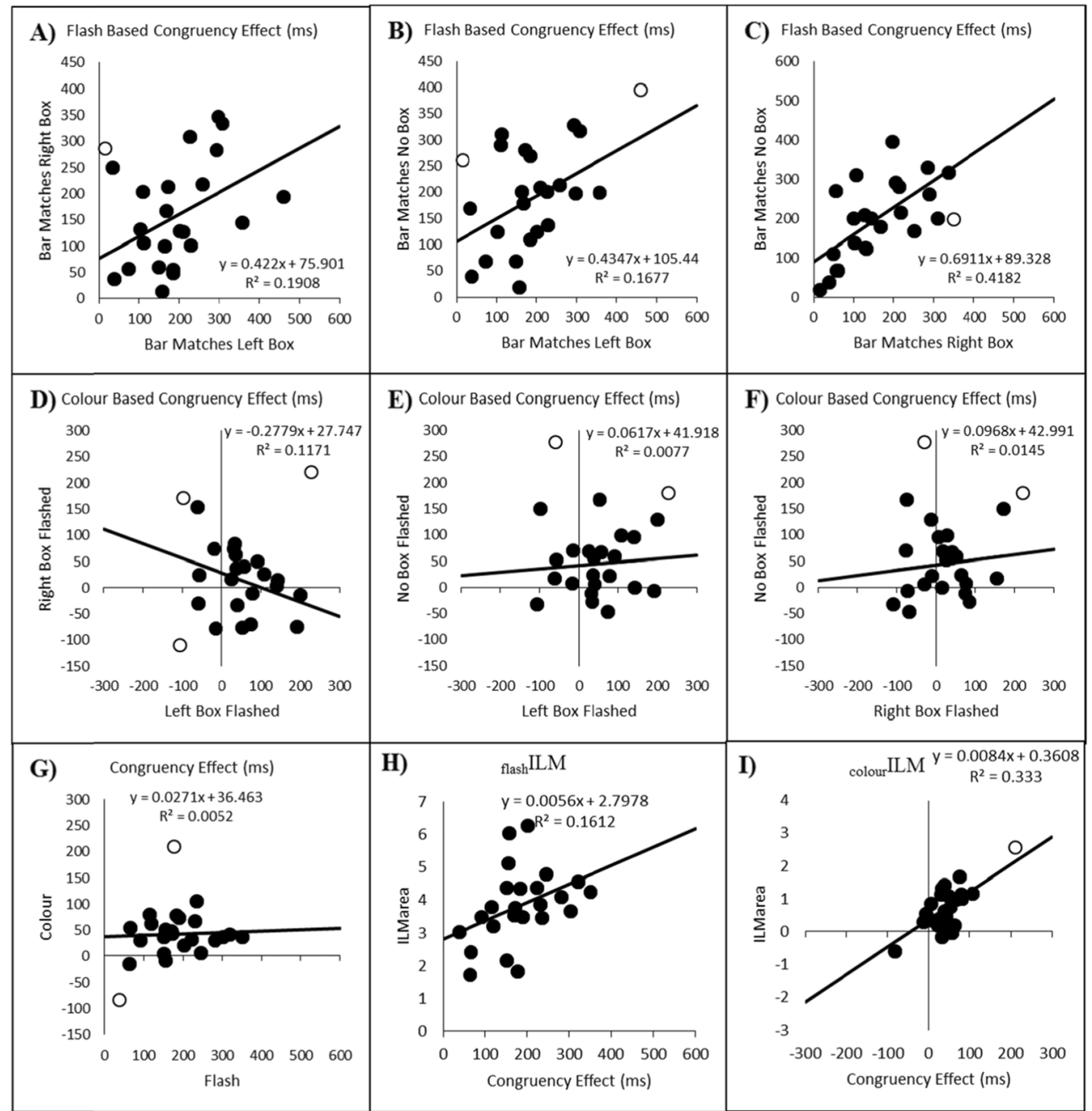

Fig. 7 Correlations between decision time congruency effects for a-c flash ILM, d-f colourILM, $\mathbf{g}$ between flash ILM and colour ILM, h-i with $\mathrm{ILM}_{\text {area }}$ for flash and colour, respectively. Conditions a and $\mathbf{d}$ show flash ILM and colour ILM when controlling for a left inducer of colour ILM and ${ }_{\text {flash }}$ ILM, respectively. Conditions $\mathbf{b}$ and $\mathbf{e}$ show flash ILM and

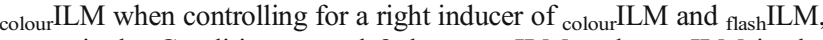
respectively. Conditions $\mathbf{c}$ and $\mathbf{f}$ show flash ILM and colour ILM in the absence of an inducer of colour ILM and flash ILM, respectively. Each data point represents a participant; open symbols indicate participants dropped from analysis because they exceeded Cook's distance criterion 


\section{Discussion}

The results of Experiment 1 replicated the high test-retest

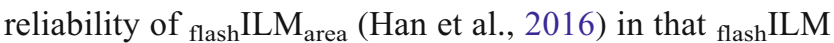
when the bar colour matched the left box was correlated with flash ILM when the bar colour matched the right box. Moreover, flashILM during trials where the bar colour matched a box was correlated with flashILM when the bar colour did not match a box. Finally, there was no evidence that the size of flashILM was influenced by whether or not colourILM was present. The colourILM was related between mixed and pure trials, although the expected relationship between colourILM in the context of left and right flash trials was not found. The Bayesian analysis of the data in this later case, however, showed the data to be equivocal, and could be considered equally supportive of both the null hypothesis and the alternative. It was reasoned that if the relationship was not present, then averaging two unrelated values should not have resulted in a relationship with the pure colourILM condition, but if the relationship is present, then averaging should improve the estimate and therefore assist in revealing any relationship. Given the latter was found, the equivocal finding is interpreted as indicating that the relationship is present, but it appears that it is less robust than that for flash ILM. This may reflect the fact that colourILM appears to be smaller than flashILM in this experiment, although the size of the colourILM was not influenced by the presence of flashILM. Finally, while ILM in both cases was larger if participants responded towards the inducing box, response instruction did not interact with ILM type, and ILM was present when responding away from the inducing box, indicating that neither flashILM nor colourILM is a result of simply choosing to respond towards (or away from) the inducing stimulus.

In addition, the decision times also provided a measure by which ILM could be investigated, namely the decision time congruency effect. While Han et al. (2016) did not find this measure to be correlated with ILM $_{\text {area }}$, the current data set did suggest both the percept score and decision times were being influenced by a common underlying cause, presumably the illusory motion. While the relationship between the decision time congruency effect and ILM $_{\text {area }}$ fell just short of significance for flash ILM, it was strong for colour ILM. A comparison between these correlations, however, did not find them to be different from each other, and so there was no evidence to suggest an interaction. Therefore, the data is interpreted as showing a moderate correlation for both types of ILM. There was no relationship between an individual's decision type congruency effect for flash ILM and colour ILM, suggesting the two illusions are unrelated in how they arise, but the motion signal they create influences decision speed similarly. Because both forms of ILM speed responding as the illusion gets bigger, but there is no correlation between the two congruency effects, it suggests that the decision time congruency effect arises post-motion generation and reflects response related aspects of the tasks but the motion itself arises for separate and independent reasons.

In support of this interpretation was the fact that ${ }_{\text {flash }}$ ILM and colour ILM were not related to each other, and the data was not equivocal with the Bayesian analysis showing positive evidence in support of the null hypothesis. This lack of a relationship between flash ILM and colourILM further suggests the illusions arise for independent reasons (Han et al., 2016). While flash ILM could arise due to exogenous visual attention, such an explanation is not tenable for colourILM. However, colour ILM is thought to reflect attribute priming (von Grünau \& Faubert, 1994) or TAM (Tse et al., 1998). TAM is thought to reflect the object system interpreting the new display as containing no new objects, but rather a transformation of an existing object. When the bar matches based upon colour, the display is interpreted as the matching box stretching and becoming the bar, and this results in motion being detected. In the ${ }_{\text {flash }}$ ILM display, however, the bar matches the nonflashed box, and as such this constant object tracking explanation does not apply. Therefore, the current results from both the percept scores and the decision times are consistent with the separation of these illusions, each of which requires a separate explanation and therefore are different phenomenon.

Attribute priming resulting in TAM could also account for the shape based ILM (shapeILM ; Corballis et al., 2002; Tse et al., 1998). It has been shown that presentation of a thick or thin bar will tend to be away from a box with a height that matches the thickness of the bar (Corballis et al., 2002; Tse et al., 1998). In addition, shape ILM was shown to be limited to

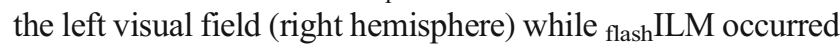
in both visual fields (both hemispheres). Corballis et al. (2002) suggested that the two illusions reflected the operation of different underlying mechanisms, consistent with the notion that flash ILM may reflect the attentional gradient and colour ILM may reflect TAM.

The conditions that produce shape ILM and colourILM are similar in that the boxes differ in a noninformative way, and the presentation of the bar is such that it corresponds to one of the boxes in the relevant dimension. Finally, illusory motion is reported away from the matching box. While the matching is based upon different physical attributes (shape vs. colour), ILM has been shown to operate across various visual dimensions (von Grünau \& Faubert, 1994), and so these two forms of attribute priming may reflect a common underlying process based upon interpreting the change in the display as reflecting a change in an object within the display (TAM) rather than as the appearance of a new object. Because TAM is thought to operate at the object representation level and not low-level visual features, then despite the differences in the attributes, one would expect those who show a large colourILM to also 
show a large shape $\mathrm{ILM}$. This is because TAM is the inclusion of motion based upon tracking of an object regardless of how the object is defined. There is no reason to expect these forms of ILM to be related to the participant's flash ILM. This would be consistent with the suggestion from Experiment 1 that colour ILM and flash ILM arise for different reasons, and for the suggestion that ${ }_{\text {shape }} \mathrm{ILM}$ and ${ }_{\text {flash }} \mathrm{ILM}$ also arise for different reasons (Corballis et al., 2002; Han et al., 2016).

\section{Experiment 2}

In Experiment 2, colour ILM, shape ILM, and ${ }_{\text {flash }}$ ILM trials were intermixed in a single block of trials to determine if the inde-

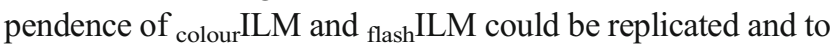
determine if colour ILM and shape $\mathrm{ILM}$ are related to a common underlying mechanism that is responsible for attribute priming, which is currently thought to be object representations (Tse et al., 1998).

\section{Method}

\section{Subjects}

Twenty-four students (10 males, 14 females; $M_{\text {age }}=19.83$ years, $S D=2.14$ ), recruited from The University of Auckland student body, volunteered to participant in this study. All reported normal or corrected-to-normal vision, and 21 were right-handed, as assessed by a score $>0(M=$ 79.50), and three were left-handed, as assessed by a score $<0$ $(M=-42.1)$, on the Edinburgh Inventory (Oldfield, 1971). All methods were approved by the University of Auckland's Human Participants Ethics Committee.

\section{Apparatus}

The same apparatus was used for stimulus presentation and data collection as in Experiment 1.

\section{Stimuli}

The background was a neutral grey $\left(13.07 \mathrm{~cd} / \mathrm{m}^{2}\right)$. The fixation cross was presented in black $\left(4.63 \mathrm{~cd} / \mathrm{m}^{2}\right)$ in the centre of the display and measured $0.53^{\circ} \times 0.53^{\circ}$. There were three categories of trial types, namely colour ILM trials, shape ILM trials, and flash ILM trials. On colour ILM and ${ }_{\text {flash }}$ ILM trials the two boxes measured $1.96^{\circ} \times 1.96^{\circ}(41 \times 41=1,681$ pixels $)$, and were separated by $9.60^{\circ}$ centre to centre. The left and right boxes' centres were equally shifted left and right from the fixation cross and were $1.75^{\circ}$ above the centre of the fixation cross. The bar that joined the boxes touched the edge of both boxes and was increased to $1.96^{\circ}$ to match the box height, and it was centred with the boxes. The display is presented in

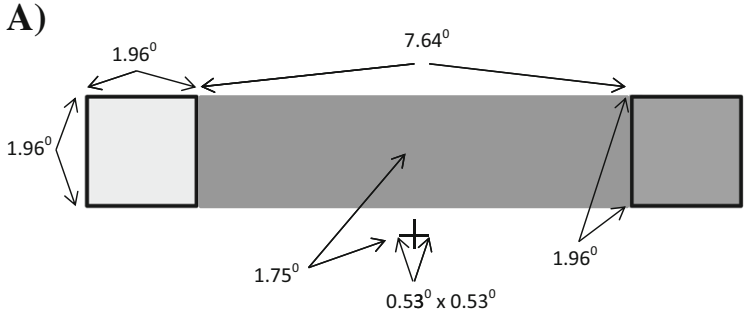

B)

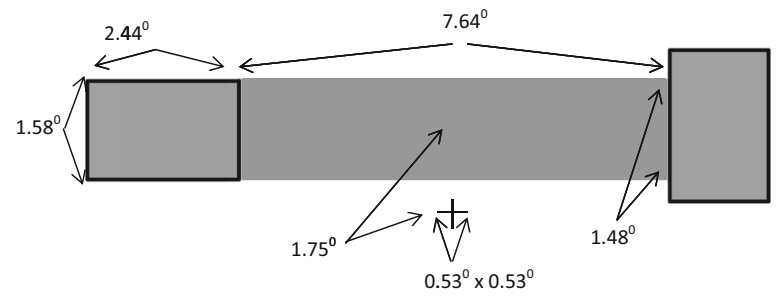

Fig. 8 a flash/ colourILM display; $\mathbf{b}_{\text {shape }}$ ILM displays

Fig. 8. Note, in the figure, the boxes are shown with visible outlines to allow them to be visible against the bar, but in the experiment, when the bar was presented, the edge was not visibly distinct from the box of the same colour on colour ILM (suggested by the different shades of grey), or from either box on flash trials. On shape ILM trials, the two boxes were of rectangular in shape $\left(1.58^{\circ} \times 2.44^{\circ} ; 33 \times 51=1,683\right.$ pixels $)$, with the major axis of one box being vertical and the other horizontal. Shape ILM trials were equally divided between left and right boxes having the major axis vertically oriented. On shape $\mathrm{ILM}$ and flash $\mathrm{ILM}$ trials the boxes and bar were all a neutral grey $\left(7.68 \mathrm{~cd} / \mathrm{m}^{2}\right)$.

\section{Procedure}

Minimizing luminance differences The luminance differences between the starting red, green, and grey were minimized using the same flicker procedure as Experiment 1 . This resulted in the following luminance settings, red 7.85 $\mathrm{cd} / \mathrm{m}^{2}$, green $7.70 \mathrm{~cd} / \mathrm{m}^{2}$, and grey $7.68 \mathrm{~cd} / \mathrm{m}^{2}$. The luminance of the grey flash was 23.76 .

Trial sequence A trial began with the presentation of the fixation cross for $500 \mathrm{~ms}$, after which the two boxes were presented for $500 \mathrm{~ms}$. On flash trials, either the left or right box increased in luminance for $50 \mathrm{~ms}$, while on colour and shape trials, no change occurred during this interval. On the same frame that a flashed box would return to its initial luminance value the two boxes were joined by the bar based upon one of the seven levels of real motion. On flash and colour trials the bar was the same height as the two boxes, while on half of the shape trials the bar matched the height of the taller box, and on half of the trials the bar matched the height of the shorter box. On half of the colour trials the bar was red, and on 
the other half the bar was green, with the luminance matching the respective boxes. On shape and flash trials, the bar was the same neutral grey as the boxes. There were 140 trials of shape ILM, colourILM, and flash ILM, equally distributed over the seven levels of real motion and left and right side inducer, for a total of 420 trials.

Half the participants were required to respond by indicating which direction the bar appeared to move, meaning at which square did the motion terminate, and half were to respond by indicating which square the motion began, or where did the bar come from. Responses that indicated the bar moved to the left (came from the right) were scored as -1 , and responses that indicated the bar moved to the right (came from the left) were scored as +1 . All trials responded to faster than $200 \mathrm{~ms}$ $(0.17 \%)$ were deemed to be anticipations and those responded to longer than $2,000 \mathrm{~ms}(5.55 \%)$ were deemed to be distractions and were discarded. Trials in which no response was made $(0.21 \%)$ were also discarded. The average of the remaining "percept scores," and decision times were then calculated for analysis from the remaining trials $(94.08 \%$ of the 10,080 trials run over all participants).

\section{Results}

The percept scores for the ${ }_{\text {flash }} \mathrm{ILM}$, colour ILM, and shape ILM can be seen in Fig. 9. The area between the curves was calculated for ${ }_{\text {flash }}$ ILM, colour ILM, and shape ILM and can be seen in Fig. 10. The percept scores were analysed in a one-way ANOVA with ILM type as the factor (colour ILM, shape ILM, and ${ }_{\text {flash }}(\mathrm{LM})$. This resulted in a main effect of ILM type, $F(2,46)=4.05, p=.024, M S E=2.021, \eta^{2}=0.150 ; M=$ 3.296, 3.031, and 4.149, for colourILM, shape ILM, and flash ILM, respectively. Planned contrasts were used to compare colour ILM and shape ILM to each other, which did not differ, $F(1,23)=1.74, p=.200, M S E=0.972, \eta^{2}=0.070$, and to compare ${ }_{\text {flash }}$ ILM to the average of colour ILM and shape ILM, which showed flash $_{\text {ILM }}$ to be larger, $F(1,23)=4.37, p=.048$, $M S E=21.339, \eta^{2}=0.160$.

\section{Relationships between ILM types}

After discarding data pairs that exceeded the inclusion criterion (Cook's distance $<=0.1818 ; 4 /(n-k-1)$ where $n=24$ and $k$ $=1$ ), an individual's ILM $_{\text {area }}$ for the two attribute priming conditions were found to be related, $r^{2}(22)=.550, p<.001, \mathrm{p}_{\mathrm{H} 0 \mid \mathrm{D}}$ $<0.001$, very strong evidence against the null hypothesis. In contrast, neither colourILM, $r^{2}(19)=.013, p=.620, \mathrm{p}_{\mathrm{H} 0 \mid \mathrm{D}}=$ 0.799 ; nor shape $\mathrm{ILM}, r(19)=.003, p=.816, \mathrm{p}_{\mathrm{HO} \mid \mathrm{D}}=0.8163$, both positive evidence in favour of the null hypothesis, was related to flash ILM. If the extreme data pairs are included then the correlation between colour ILM and $_{\text {flash }} \mathrm{ILM}$ is $r^{2}(22)=.012$ and for shape $\mathrm{ILM}$ and ${ }_{\text {flash }} \mathrm{ILM} r^{2}(22)=0.0004$. The scatter plots are shown in Fig. 11.

\section{Decision times}

The decision times are shown in Fig. 12. As in Han et al. (2016), the group mean decision times were then plotted as a function of the distance from the point of subjective equality and were fitted to a distance decay function of the form $\mathrm{dt}=\mathrm{dt}_{\mathrm{a}}$ $+\mathrm{dt}_{0} \mathrm{e}^{-\mathrm{rx}}$, where $\mathrm{dt}_{\mathrm{a}}$ is a lower bound asymptote, $\mathrm{dt}_{0}$ is the amount above $\mathrm{dt}_{\mathrm{a}}$ that is predicted at the PSE, $r$ reflects the rate of decay, and $x$ is the distance at which to evaluate the function. The decision times showed a good fit to the distance decay function $\mathrm{dt}=477.7509+413.42808 \mathrm{e}^{-0.3051 \mathrm{x}}, r^{2}(39)=$ $.7995, p<.001, \mathrm{p}_{\mathrm{H} 0 \mid \mathrm{D}}<0.001$, very strong evidence against the null hypothesis. Note, the 14 decision times from colour ILM, shape $\mathrm{ILM}$, and ${ }_{\text {flash }} \mathrm{ILM}$ were all well described when a distance decay function was fitted to each conditions separately, with all $r^{2}$ values greater than 0.73 .

\section{Decision time congruency affect}

The decision time congruency effect was calculated for each ILM type and analysed in a one-way ANOVA, with ILM type (colour $\mathrm{ILM}$, shape $\mathrm{ILM}$, and flash $\mathrm{ILM}$ ) as the factor. This revealed no difference in the magnitude of the congruency effect between conditions, $F(2,46)=0.395, p=.676, M S E=$ 6968.141, $\eta^{2}=0.017, M=205,198,184 \mathrm{~ms}$ for colour ILM,

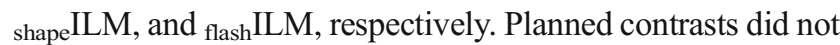
reveal any difference between the congruency effect for colour ILM and shape ILM, $F(1,23)=0.331, p=.571, M S E=$ $4323.398, \eta^{2}=0.014$, nor between the average of colour $\mathrm{ILM}$

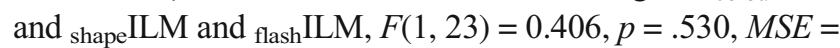
$70647.501, \eta^{2}=0.017$.

\section{Relationships between congruency effects}

The congruency effects were related between colour ILM and shape ILM, $r^{2}(22)=.654, p<.001, \mathrm{p}_{\mathrm{H} 0 \mid \mathrm{D}}<0.001$, very strong evidence against the null hypothesis, while the flash $_{\text {ILM con- }}$ gruency effect was not correlated with the congruency effect from either the colour ILM, $r^{2}(22)=.0008, p=.897, \mathrm{p}_{\mathrm{H} 0 \mid \mathrm{D}}=$ 0.829 , positive evidence in favour of the null hypothesis, or shape $\mathrm{ILM}, r^{2}(21)=.0007, p=.906, \mathrm{p}_{\mathrm{H} 0 \mid \mathrm{D}}=0.826$, positive evidence in favour of the null hypothesis. If the point that exceeded the inclusion criterion (Cook's distance $<=0.1818$ ) for shape ILM is included then the $r^{2}(22)=.0082$. The scatterplots are shown in Fig. 13.

\section{Relationship between congruency effect and ILM area}

As shown in Fig. 14, the congruency effect was correlated with ILM $_{\text {area }}$ from the corresponding colour ILM, shape ILM, and flash $\mathrm{ILM}$ conditions to determine if the two measures were tapping into a common underlying construct. After removing data pairs that exceeded the inclusion criterion (Cook's distance $<=$ 

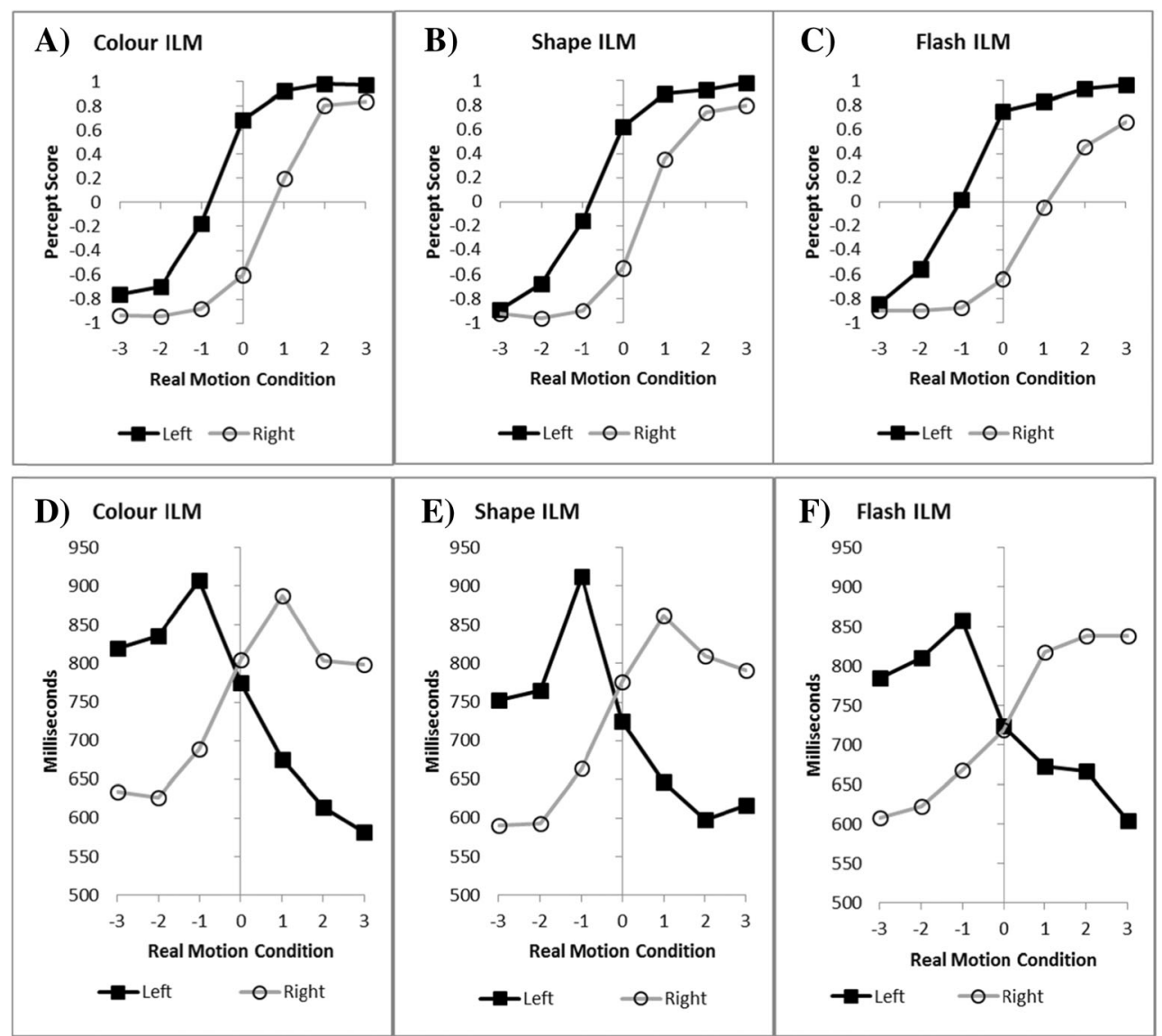

Fig. 9 Upper panels depict group mean percept scores; lower panels depict group mean decision times as a function of inducer location and real motion. Left columns show data from colourILM conditions. Middle columns show data from shape ILM conditions. Right columns show data from ${ }_{\text {flash }}$ ILM conditions
0.1818 ), there was a relationship between the congruency effect

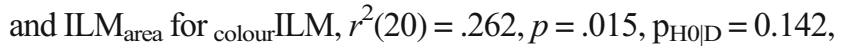
positive evidence against the null hypothesis, and ${ }_{\text {flash }} \mathrm{ILM}$, $r^{2}(19)=.491, p<.001, \mathrm{p}_{\mathrm{HO} \mid \mathrm{D}}=0.004$, very strong evidence against the null hypothesis, conditions, but not for the shape ILM, $r^{2}(21)=.031, p=.419, \mathrm{p}_{\mathrm{H} 0 \mid \mathrm{D}}=0.769$, positive evidence in favour of the null hypothesis, condition. While the correlation found for ${ }_{\text {flash }} \mathrm{ILM}$ was stronger than that for

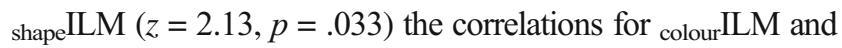
shape ILM did not differ $(z=1.21, p=.227)$. Given the difference between ${ }_{\text {flash }}$ ILM and shape ILM would not survive a post hoc correction, it is suggested the results do not offer strong evidence towards suggesting the influence of motion on the congruency effect differs between ILM types. If the extreme points are left in

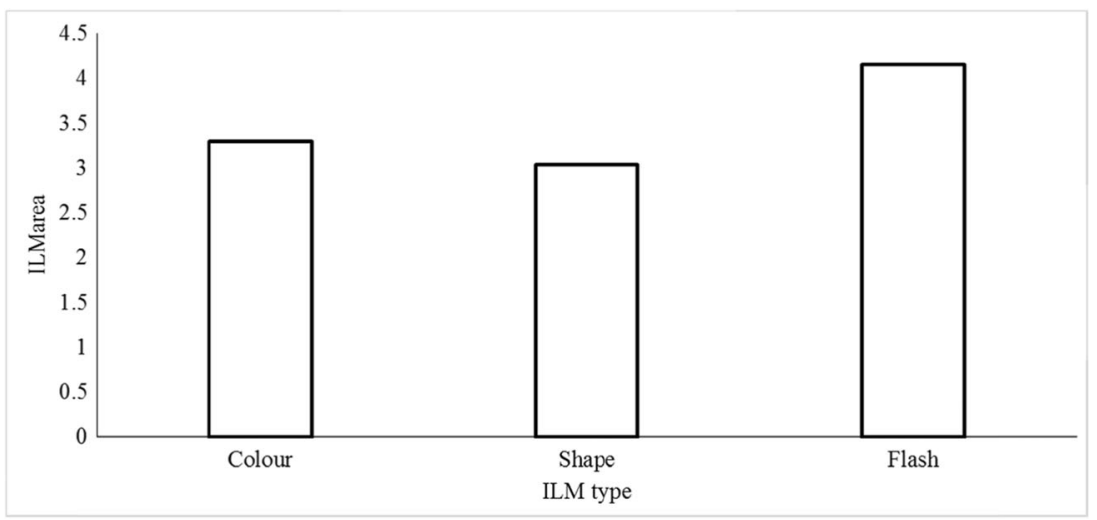

Fig. 10 Group mean ILM area for colour ILM, shape ILM, and flash ILM 


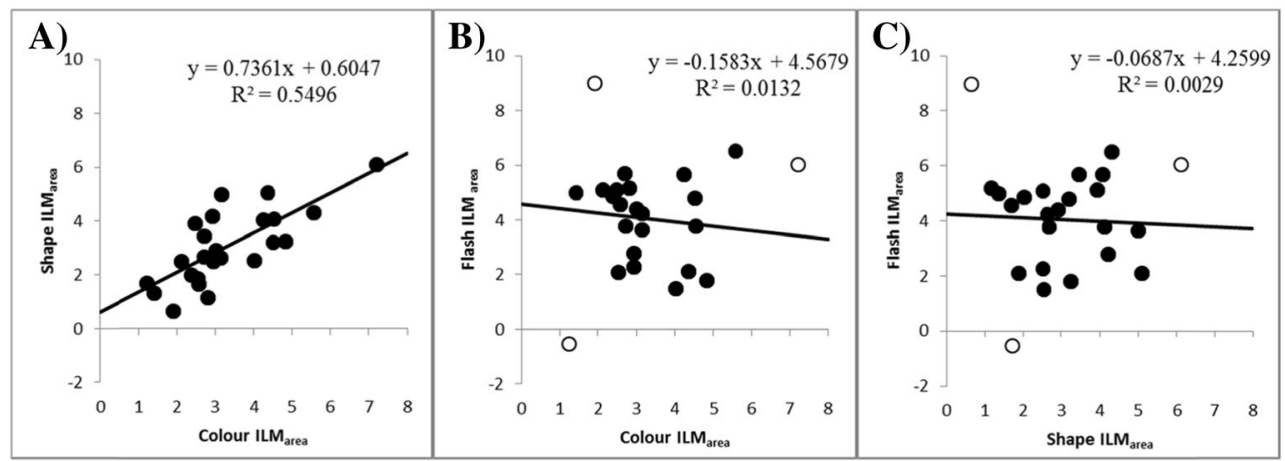

Fig. 11 Correlation between ILM area $_{\text {for }} \mathbf{a}_{\text {colour }} \mathrm{ILM}$ and shape $\mathrm{ILM}, \mathbf{b}_{\text {colour }} \mathrm{ILM}$ and $\mathrm{flash}_{\mathrm{ILM}} \mathrm{IL}$, and $\mathbf{c}_{\text {shape }} \mathrm{ILM}$ and flash $\mathrm{ILM}$. Each data point represents an individual participant; open symbols indicate data points excluded from analysis due to exceeding Cook's distance criterion

the correlations are $r^{2}(22)=.331, .108$, and .494 , for colour ILM, shape ILM, and flash $I L M$, respectively.

Comparison of flash ILM and colour ILM between Experiments 1 and 2

Given that ILM $_{\text {area }}$ for Experiment 1 was calculated only for a motion range of -2 to +2 , while in Experiment 2 ILM $_{\text {area }}$ is calculated over a motion range of -3 to +3 , the area values are not directly comparable. Therefore ILM $_{\text {area }}$ for Experiment 2 was recalculated over the -2 to +2 range, resulting in mean values for colour $\mathrm{ILM}_{\text {area }}$, shape $\mathrm{ILM}_{\text {area }}$, and flash $\mathrm{ILM}_{\text {area }}$ of 2.93, 2.69, and 3.56, respectively. The ${ }_{\text {flash }} \mathrm{ILM}_{\text {area }}$ from Experiment 1 was 3.80 averaged over all participants. The ${ }_{\text {flash }} \mathrm{ILM}_{\text {area }}$ values from Experiment 2 do not significantly differ from those of Experiment $1 t(46)=.62 p=.54$. In contrast, colour $\mathrm{ILM}_{\text {area }}$ was smaller in Experiment 1 than Experiment 2, $t(46)=8.44, p<$ .001 , mean area 0.84 versus 2.93 .

The only appreciable difference was that in Experiment 2 the height of the colour bar matched the height of the inducing boxes while in Experiment 1 the bar was narrower than the height of the boxes. This appears to have accentuated

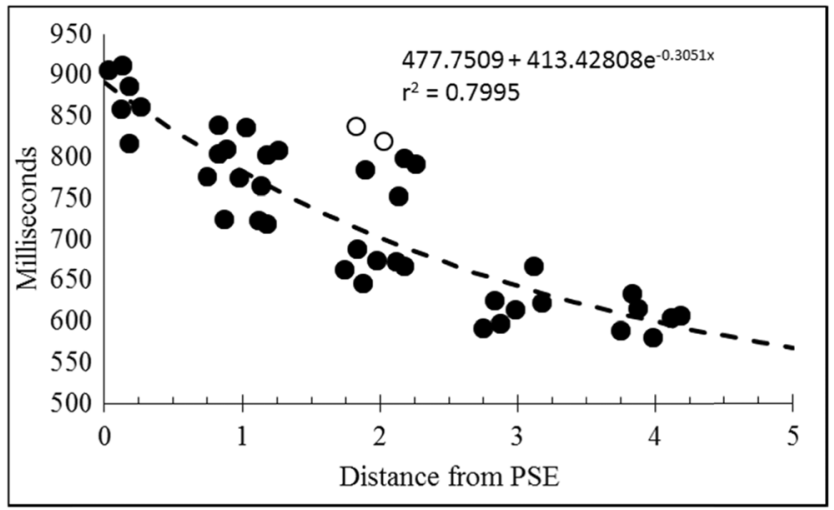

Fig. 12 Group mean decision times as a function of the distance from the mean point of subjective equality. Open symbols indicate data points omitted from analysis due to exceeding Cook's distance criterion
colourILM, while contributing no detectable change in flashILM. This influence of the correspondence between bar and box sizes on colour ILM but not flash ILM further points towards these two illusions of motion being a result of different underlying causes and further suggests a link between colour ILM and shape ILM. It is also consistent with colour ILM resulting from TAM because, while the similarity of colour would work towards linking the box and bar, the change in size would assist in the parsing of the bar into a separate object. By making the box and bar the same height, they will be more likely to be interpreted as the same object changing shape.

\section{Discussion}

All conditions produced large and robust ILM. Importantly,

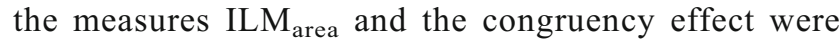
correlated between colourILM and shape ILM but not between the attribute priming conditions and the corresponding

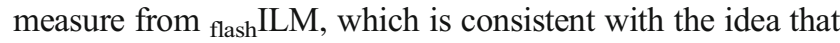
the colour and shape conditions result in a motion illusion for entirely different reasons than the flash condition. The relationship between ILM $_{\text {area }}$ and the congruency effect was not stable, with colourILM and flashILM conditions showing this relationship, while shape ILM did not, with an ambiguous result, when comparing the strength of the correlations between conditions. It should be noted that Han et al. (2016) also failed to find a relationship between the congruency effect and ${ }_{\text {flash }}$ ILM $_{\text {area }}$, although they did find a relationship between

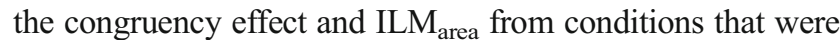
unrelated to flash ILM.

It is suggested that the congruency effect reflects post-motion effects, where large motion signals result in faster decision times. This is consistent with the finding that the decision times themselves show an exponential distance decay function from the point of subjective equality, which also suggests that slower responses are made to smaller signals as in boundary effects (Cartwright, 1941). The commonality is in that all illusions are illusions of motion, and both TAM (Tse, 2006) and 

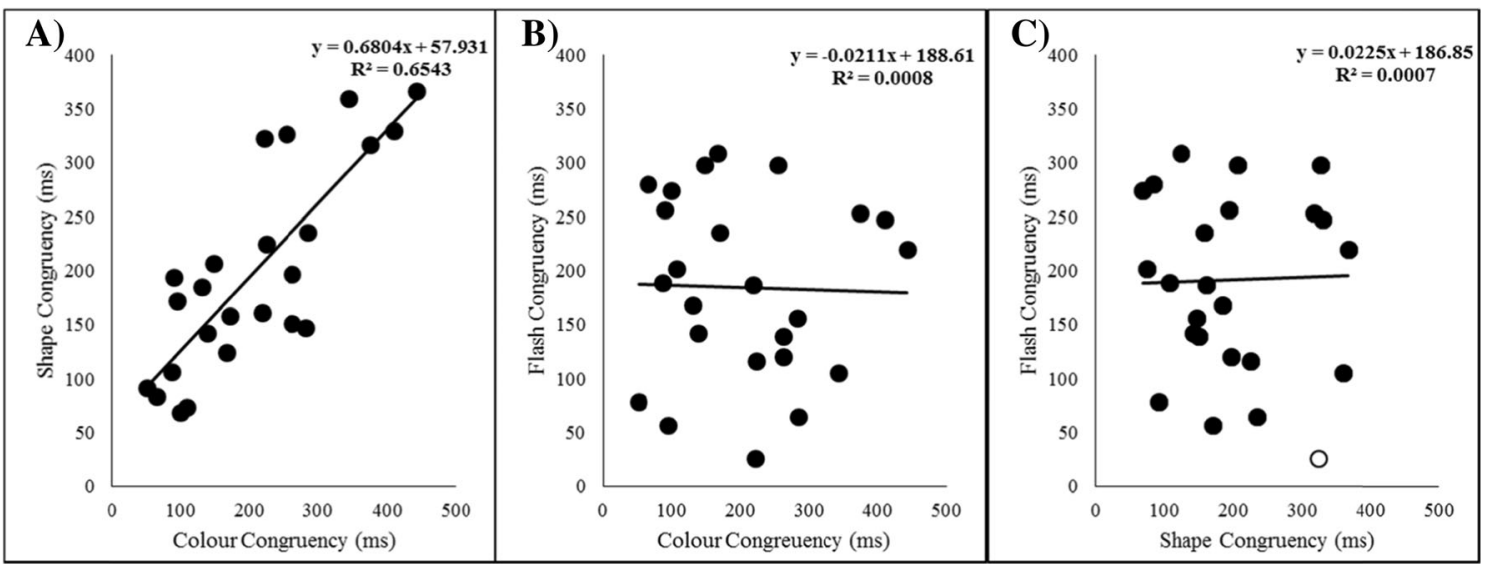

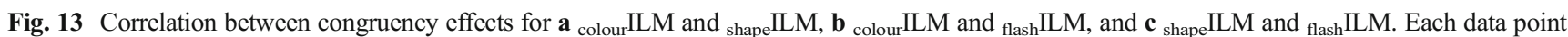
represents a participant; open symbols indicate participants dropped from analysis because they exceeded Cook's distance criterion

flash ILM (Hamm et al., 2014) activate human motion areas, but the lack of a correlation between an individual's TAM and flash ILM suggests independence of how the motion signal is generated.

The flash ILM paradigm is known to activate the neural networks associated with visual attention (Hamm et al., 2014). ILM can also be generated based upon cross modal orienting of attention (Shimojo et al., 1997) or by reflexive gaze orienting (Bavelier et al., 2002). Ha et al. (in press) have

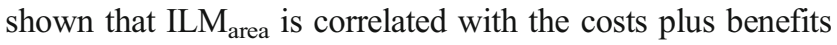
from exogenous cuing. Therefore, multiple lines of evidence converge upon the interpretation that flash ILM is primarily reflective of a gradient of exogenous visual attention.

Given that Experiment 2 showed that colourILM and shape ILM were related to each other, and given that both Experiment 1 and 2 failed to show any evidence suggesting colourILM is related to flash ILM, it is concluded that neither colourILM nor shape ILM arise due to visual attention, but that they are different illusions of motion from flashILM. This finding lends empirical support to the notion that attention priming and attribute priming are generating illusions of motion through separate underlying systems (Corballis et al., 2002).

Both colour ILM and shape ILM take the form of motion away from the box that matches the bar on the relevant attribute. This is consistent with the function of an impletion process suggested by Downing and Treisman (1997), where the display is considered improbable because objects do not suddenly appear but rather emerge in motion. Impletion is suggested to work to create the most real-world plausible interpretation of the display. So inferring motion and then binding it to a matching preexisting stimulus is highly consistent with this notion. However, given the decision times get slower as one approaches the point of subjective equality it appears that real motion and illusory motion cancel the motion signal (see Crawford et al., 2010; Han et al., 2016), which implies a plausible real-world display is now being made implausible. Given that the real motion is not ambiguous, ILM resulting from a process that makes the display more realistic by adding motion should not then make the display less realistic by cancelling existing motion. Moreover, simply replacing the term attribute priming with impletion does not provide greater
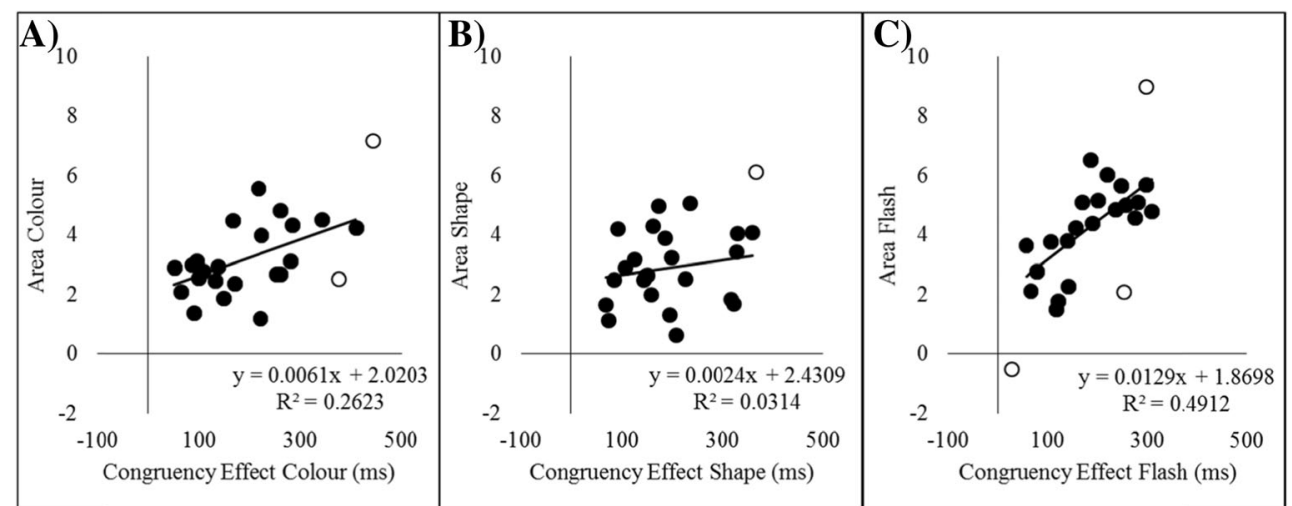

Fig. 14 Correlations between decision time congruency effect and ILM $_{\text {area }}$ for $\mathbf{a}_{\text {colour }}$ ILM, $\mathbf{b}_{\text {shape }}$ ILM, and $\mathbf{c}$ flash ILM. Each data point represents a participant; open symbols indicate participants dropped from analysis because they exceeded Cook's distance criterion 
insight into how impletion, or how attribute priming, might be accomplished.

Jancke et al. (2004) suggested that ILM results from sub-threshold activity radiating outwards from the inducing stimuli. This activity speeds the detection of the bar near the box, similar to the attentional gradient explanation. If we presume this subthreshold activity could be working along colour specific channels then one could explain colourILM through such a mechanism. However, shape ILM cannot be explained this way since the increased spreading of activity should always be greater from the larger edge of the tall box, and so ILM should always be away from the tall box and not from box that matches the size of the bar. Given the results suggest colourILM and shape ILM reflect a common underlying system generating the illusions; since this does not work for shape ILM, then it follows that it is not the explanation for colourILM. It should be noted that spreading subthreshold activity also predicts ILM towards the flash when an existing bar is removed, but ILM in this case is still away from the flash (Crawford et al., 2010; Hamm et al., 2014; Han et al., 2016). And since onset bars and offset bars produce ILM that his highly correlated (Han et al., 2016), this explanation is rejected for flash ILM as well. It should be noted that while this explanation is rejected as the primary explanation, it is entirely possible that subthreshold spreading of activation occurs and has some influence on the final percept. It seems quite unlikely that the perception of motion is based upon a single mechanism.

One possible explanation that could explain both colour ILM and shape ILM may be the contrast counterchange explanation suggested by Hock and Nichols (2010), although some expansion of the notions would be required. The basic idea behind the contrast counterchange explanation is that when the bar onsets there are regions where the luminance contrast decreases, such as the edge between the matching box and the bar, and regions where luminance contrast increases, between the bar surface and the background. In the colour condition, the onset of the bar and the mismatching coloured box continue to show a contrast in hue, even though the luminance contrast would have reduced at both ends of the bar due to the minimizing of luminance differences based upon flicker fusion. Also, because the colourILM condition counterbalances hue, even if one colour were brighter than the other, the left and right percept curves include both red and green hues and so the conditions do not vary with colour or luminance. In the contrast counterchange explanation for ILM, motion is perceived in the direction from a decrease in luminance contrast towards an increase in luminance contrast. And since the luminance contrast would decrease at both ends of the bar, and increase between the bar surface and the background, the result should have been motion towards the centre, not directional motion. If we allow for the contrast changes to be in terms of contrasting hues, then this fits with motion away from the matching coloured box as the edge with the bar disappears, towards the bar surface, with the nonmatching edge coded as an increase in contrast in colour space as the grey background would be relatively central in colour space. Therefore, the suggestion is that contrast counterchange may operate based upon contrast in dimensions additional to luminance.

When shape ILM is considered in terms of spatial frequency, the loss of the edge between the bar and the box results in a decrease in terms of activity in the high spatial frequencies. At the mismatching edge there is an always an increase in the high spatial frequencies, due to the short segments that are created at the mismatching box, as shown in Fig. 15. If the reduction in high spatial frequency activity at the matching box is coupled with the shift to higher spatial frequencies at the mismatching box acts in the same way as decreases and increases in contrast, then this may account for the shape ILM. Therefore, ILM in both the colour and shape attribute priming conditions may reflect the sensitivity of a participant's motion system to these nonluminance forms of contrast counterchange. It should be noted that von Grünau and Faubert (1994) have shown that ILM can be defined along various channels, such as luminance, hue, texture, stereo depth, and so forth, but that motion will result when the box and bar are defined by different attributes, and so the motion can be induced cross modally.

The simplest explanation for the attribute priming conditions is therefore based on the fact that these conditions are where one of the boxes can be interpreted as having moved because it changed shape, which is known as transformational apparent motion (Tse et al., 1998). The edge along the matching box and the bar both result in the loss of a boundary between the box and the bar. In Experiment 2 this was much more pronounced than Experiment 1 for the colourILM because the bar was now the same height as the square. In Experiment 1, the boundaries between the box and the bar were still apparent due to the points of deep concavity which would operate against the interpretation of the bar being the box after elongation. The magnitude of the effect was larger in Experiment 2 when the bar was not easily parsed into a separate object from the box and so the display is more easily interpreted as an object changing shape. The size of flashILM, however, did not differ based upon whether or not the bar was easily parsed into a separate object, suggesting that ${ }_{\text {flash }}$ ILM and colourILM are sensitive to different manipulations.

Sudden changes in shape of an existing object has been shown to be interpreted as the object changing in shape in motion over time (Chen \& Scholl, 2016; Tse et al., 1998), which 


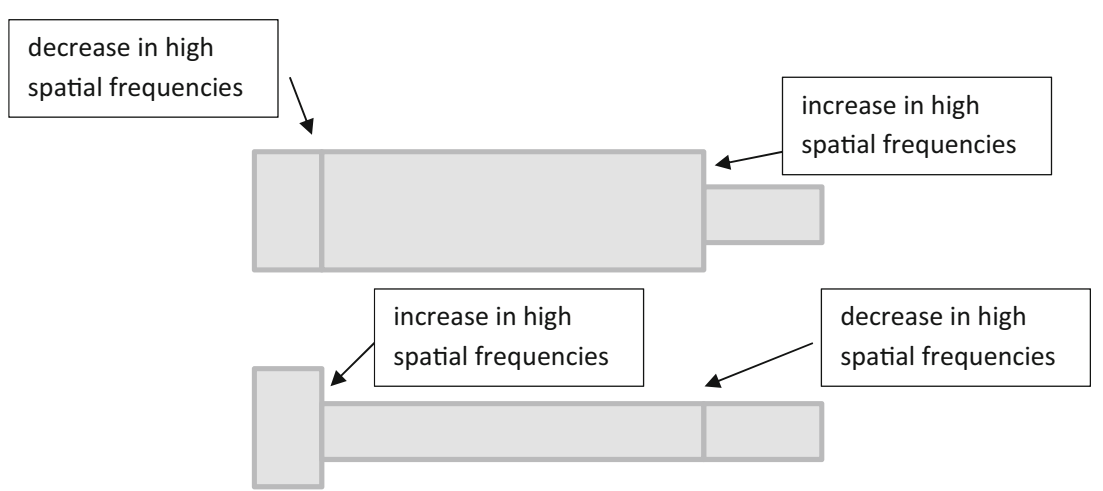

Fig. 15 Decreases and increases in spatial frequency during shape ILM presentations. During experiment, the bar was indistinguishable from the boxes; outlines are for clarification purposes only

corresponds to the situations comprising the attribute priming conditions. The attribute priming conditions produce motion from the matching box towards the non-matching box. Consistent with this suggestion is the fact that the neural networks associated with transformational apparent motion, namely motion and object areas (Tse, 2006), are markedly different from the motion and attentional networks found flash ILM (Hamm et al., 2014), with only hMT+ being common.

While the attentional gradient explanation for ${ }_{\text {flash }} \mathrm{ILM}$ struggles to explain colour ILM and shape $\mathrm{ILM}$, the TAM explanation struggles to account for flash ILM where the box that most closely matches the bar is the nonflashed box; the flashed box is brighter than the bar when it flashes while the nonflashed box is of the same luminance as the bar. In other words, flash ILM is characterised as motion away from the nonmatching box towards the matching box while colour $\mathrm{ILM}$ and shape ILM are characterised as motion away from the matching box towards the nonmatching box, which makes it harder to group into a common explanation. Given the individual correlational results of both Experiment 1 and Experiment 2 suggest that ${ }_{\text {flash }} \mathrm{ILM}$ is unrelated to colour ILM and shape ILM, explaining flash ILM by the attentional gradient and colour ILM and shape ILM by TAM is entirely consistent with the data. Not only is the explanation consistent with the data, it also starts to organise the findings in the literature into different groups, or clusters, of illusions that may reflect different underlying causes and therefore do not inform particularly well theories based upon findings from different paradigms.

The ILM literature appears to be a rich source of findings that may inform us on a number of different processes that process visual information in ways that serve to enhance our perception of motion; there may be many different processes that, when grouped together, describe how impletion (Downing \& Treisman, 1997) is accomplished. This suggests that impletion is not itself a process, but rather is a description of the function of a number of separate processes, one of which may be attention and another may be transformational apparent motion.
It should be noted that the individual differences correlational approach is able to detect situations where the final percept is the result of multiple independent inputs. To demonstrate, if from Experiment 1 we were to take the left flash from the line matches left box condition and pair it with the right flash when line matches right box condition, then the area between the curves would represent the combination of both flash ILM and colourILM, which the analysis from both experiments have shown are independent of each other. We should then find that this combined $\mathrm{ILM}_{\text {area }}$ should be related to the "pure" versions of flash and colour $\mathrm{ILM}_{\text {area }}$ and that both contribute independently. When explored using a stepwise multiple regression analysis, with $p<.05$ for entrance and $p>.1$ for removal, both pure flash ILM and pure colour ILM were entered when modelling the combined version $\left(\right.$ ILM $_{\text {area }}=0.90$ flash $\mathrm{ILM}_{\text {area }}+0.43$ colour $\left.\mathrm{ILM}_{\text {area }}+0.762\right) ; r^{2}(21)=.821$, $p<.001, \mathrm{p}_{\mathrm{H} 0 \mid \mathrm{D}}<0.001$, very strong evidence against the null. In other words, using individual differences and a regression approach to the analysis of ILM data can detect multiple independent contributions to the final data, such as the combination of the flash ILM and colourILM. Obviously, in order to employ this analysis one requires relatively pure measures of the two separate contributions, and higher level interactions may exist, but all of these are empirical questions and complications that accompany any experimental approach and analysis. While it is not suggested that this approach is the panacea for all issues, this method of investigation may help to organise the existing literature into experimental protocols that are testing similar and unrelated underlying psychological processes that all result in illusory perceptions of motion.

Author note I would like to thank the action editor, Dr Wendy Adams, and two anonymous reviewers for their helpful and constructive comments that helped to improve this article. 


\section{References}

Bavelier, D., Schneider, K. A., \& Monacelli, A. (2002). Reflexive gaze orienting induces the line-motion illusion. Vision Research, 42, $2817-2827$

Briand, K. A., \& Klein, R. M. (1987). Is Posner's "beam" the same as Treisman's "glue"?: On the relation between visual orienting and feature integration theory. Journal of Experimental Psychology: Human Perception and Performance, 13(2), 228-241.

Cartwright, D. (1941). Relation of decision time to the categories of response. American Journal of Psychology, 54, 174-196.

Chen, Y. C., \& Scholl, B. J. (2016). The perception of history: Seeing causal history in static shapes induces illusory motion perception. Psychological Science, 27(6), 923-930. doi:10.1177 /0956797616628525

Christie, J. (2014). Illusory line motion is not caused by objectdifferentiating mechanisms or endogenous attention. Quarterly Journal of Experimental Psychology, 67(12), 2293-2300. doi:10.1080/17470218.2014.918633

Christie, J., \& Klein, R. M. (2005). Does attention cause illusory line motion? Perception \& Psychophysics, 67(6), 1032-1043.

Cohen, J., \& Cohen, P. (1983). Applied multiple regression/correlation analysis for the behavioral sciences (2nd ed.). Hillsdale: Erlbaum.

Corballis, P. M., Funnell, M. G., \& Gazzaniga, M. S. (2002). An investigation of the line motion effect in a callosotomy patient. Brain and Cognition, 48(2/3), 327-332. doi:10.1006/brcg.2001.1371

Crawford, T. J., Hamm, J. P., Kean, M., Schmechtig, A., Kumari, V., Anilkumar, A. P., \& Ettinger, U. (2010). The perception of real and illusory motion in schizophrenia. Neuropsychologia, 48(10), 3121-3127. doi:10.1016/j.neuropsychologia.2010.06.027

Downing, P. E., \& Treisman, A. M. (1997). The line-motion illusion: Attention or impletion? Journal of Experimental Psychology: Human Perception \& Performance, 23(3), 768-779.

Ha, H. Y., Li, D., Patten, B., \& Hamm, J. P. (in press). The relationship between flash based illusory line motion and visual exogenous attention. Canadian Journal of Experimental Psychology.

Hamm, J. P. (2001). Object-oriented millisecond timers for the PC. Behavior Research Methods, Instruments, \& Computers, 33(4), 532-539. doi:10.3758/BF03195412

Hamm, J. P., Crawford, T. J., Nebl, H., Kean, M., Williams, S. C., \& Ettinger, U. (2014). Neural correlates of illusory line motion. PLoS ONE, 9(1), e87595. doi:10.1371/journal.pone.0087595

Hamm, J. P., \& Klein, R. M. (2002). Does attention follow the motion in the "shooting line" illusion? Perception \& Psychophysics, 64(2), 279-291. doi:10.3758/BF03195792

Han, S., Zhu, Z., Corballis, M. C., \& Hamm, J. P. (2016). Illusory line motion in onset and offset bars. Attention, Perception \& Psychophysics, 78, 2579-2611. doi:10.3758/s13414-016-1170-Z

Heathcote, A. (1988). Screen control and timing routines for the IBM microcomputer family using a high-level language. Behavior Research Methods, Instruments and Computers, 20, 289-297.

Hikosaka, O., Miyauchi, S., \& Shimojo, S. (1993a). Focal visual attention produces illusory temporal order and motion sensation. Vision Research, 33(9), 1219-1240.

Hikosaka, O., Miyauchi, S., \& Shimojo, S. (1993b). Visual attention revealed by an illusion of motion. Neuroscience Research, 18(1), $11-18$.
Hikosaka, O., Miyauchi, S., \& Shimojo, S. (1993c). Voluntary and stimulus-induced attention detected as motion sensation. Perception, 22, 517-526.

Hock, H. S., \& Nichols, D. F. (2010). The line motion illusion: The detection of counterchanging edge and surface contrast. Journal of Experimental Psychology: Human Perception and Performance, 36(4), 781-796. doi:10.1037/A0016876

Jancke, D., Chavane, F., Naarman, S., \& Girinvald, A. (2004). Imaging cortical correlates of illusion in early visual cortex. Nature, 428 , $423-426$.

Kanizsa, G. (1979). Organization in vision: Essays on Gestalt perception. New York: Praeger.

Laberge, D. (1983). The spatial extent of attention to letters and words. Journal of Experimental Psychology: Human Perception \& Performance, 9, 371-379.

Masson, M. E. J. (2011). A tutorial on a practical Bayesian alternative to null-hypothesis significance testing. Behavior Research Methods, 43(3), 679-690. doi:10.3758/s13428-010-0049-5

Myors, B. (1999). Timing accuracy of PC programs running under DOS and Windows. Behavior Research Methods, Instruments, \& Computers, 31(2), 322-328.

Oldfield, R. C. (1971). The assessment and analysis of handedness: The Edinburgh inventory. Neuropsychologia, 9, 97-113.

Popper, K. R. (1963). Conjectures and refutations: The growth of scientific knowledge. New York: Harper \& Row.

Raftery, A. E. (1995). Bayesian model selection in social research. Sociological Methodology, 25(25), 111-163. doi:10.2307/271063

Schmidt, W. C., \& Klein, R. M. (1997). A spatial gradient of acceleration and temporal extension underlies three illusions of motion. Perception, 26(7), 857-874.

Schneider, K. A., \& Bavelier, D. (2003). Components of visual prior entry. Cognitive Psychology, 47(4), 333-366.

Shimojo, S., Miyauchi, S., \& Hikosaka, O. (1997). Visual motion sensation yielded by non-visually driven attention. Vision Research, 37(12), 1575-1580. doi:10.1016/S0042-6989(96)00313-6

Shore, D. I., Spence, C., \& Klein, R. M. (2001). Visual prior entry. Psychological Science, 12(3), 205-212.

Steinman, B. A., Steinman, S. B., \& Lehmkuhle, S. (1995). Visual attention mechanisms show a centre-surround organization. Vision Research, 35(13), 1859-1869.

Troscianko, T., \& Low, I. (1986). A technique for presenting isoluminant stimuli using a microcomputer. Spatial Vision, 1(2), 197-202.

Tse, P. U. (2006). Neural correlates of transformational apparent motion. NeuroImage, 31(2), 766-773. doi:10.1016/j. neuroimage.2005.12.029

Tse, P., Cavanagh, P., \& Nakayama, K. (1998). The role of parsing in high-level motion processing. In T. Watanabe (Ed.), High-level motion processing: Computational, neurobiological, and psychophysical perspectives (pp. 249-266). Cambridge: MIT Press.

von Grünau, M., Dube, S., \& Kwas, M. (1996). Two contributions to motion induction: A preattentive effect and facilitation due to attentional capture. Vision Research, 36(16), 2447-2457.

von Grünau, M., \& Faubert, J. (1994). Intraattribute and interattribute motion induction. Perception, 23(8), 913-928. doi:10.1068 /p230913 